\title{
ARTICLE Granulocytes act as a niche for Mycobacterium tuberculosis growth
}

\author{
Rustin R. Lovewell (iD) ${ }^{1}$, Christina E. Baer ${ }^{1}$, Bibhuti B. Mishra ${ }^{2}$, Clare M. Smith ${ }^{1}$ and Christopher M. Sassetti ${ }^{1}$
}

Granulocyte recruitment to the pulmonary compartment is a hallmark of progressive tuberculosis (TB). This process is well-documented to promote immunopathology, but can also enhance the replication of the pathogen. Both the specific granulocytes responsible for increasing mycobacterial burden and the underlying mechanisms remain obscure. We report that the known immunomodulatory effects of these cells, such as suppression of protective T-cell responses, play a limited role in altering host control of mycobacterial replication in susceptible mice. Instead, we find that the adaptive immune response preferentially restricts the burden of bacteria within monocytes and macrophages compared to granulocytes. Specifically, mycobacteria within inflammatory lesions are preferentially found within long-lived granulocytes that express intermediate levels of the Ly6G marker and low levels of antimicrobial genes. These cells progressively accumulate in the lung and correlate with bacterial load and disease severity, and the ablation of Ly6G-expressing cells lowers mycobacterial burden. These observations suggest a model in which dysregulated granulocytic influx promotes disease by creating a permissive intracellular niche for mycobacterial growth and persistence.

Mucosal Immunology (2021) 14:229-241; https://doi.org/10.1038/s41385-020-0300-z

\section{INTRODUCTION}

Infection by Mycobacterium tuberculosis $(M t b)$ can result in a diverse range of clinical outcomes. At one end of the spectrum, some individuals contain the infection through effective immune control, restricting Mtb growth and possibly even eradicating the bacteria. Conversely, individuals who fail to control Mtb replication or the subsequent tissue-damaging inflammation develop tuberculosis (TB), a disease defined by progressive bacterial replication, pulmonary necrosis, and cavitary lesions that promote the transmission of bacteria. ${ }^{1}$

The factors determining TB disease progression remain incompletely defined, but genetic evidence from both the human and mouse systems have converged on the importance of an immune network consisting of type I and type II interferons (IFN) and interleukin-1 (IL1). Mendelian defects in IFNy production or signaling cause susceptibility to mycobacterial infection in humans. ${ }^{2}$ Similarly, mice lacking any component of the IFNy pathway are profoundly susceptible to Mtb. ${ }^{3,4}$ T-cell-derived IFNY mediates its protective effect in at least two distinct ways. When activating macrophages, this cytokine can induce still uncharacterized antimicrobial pathways that control the intracellular replication of the pathogen, and it can induce nitric oxide synthase 2 (Nos2). ${ }^{5}$ While the resulting nitric oxide (NO) is often considered an antimicrobial mediator, in the context of Mtb infection the major protective effect of $\mathrm{NO}$ is due to its ability to inhibit the production of mature IL1 and prevent granulocyte infiltration. ${ }^{6}$ This inhibition of IL1 during persistent infection is critical for protective immunity, as the susceptibility of NO-deficient mice to Mtb infection is largely dependent on IL1 driven inflammation, ${ }^{6}$ and human genetic variants that increase IL1 $\beta$ expression are associated with more severe disease. ${ }^{7}$ Finally, the type I IFN response is a marker of disease progression, and counterregulates both IFNY and IL 1.8,9 Mutations of the type I IFN receptor are associated with protection from disease in both mouse models ${ }^{10-12}$ and humans. ${ }^{13}$ While the diversity of human immune responses described above is not reproduced in wild-type C57BL6 (Bl6 WT) mice, recent work indicates that susceptibility due to dysregulation of the IFN/IL1 axis can be modeled in other wellcharacterized mouse strains. For example, susceptibility in 129SvPas mice can be reversed by inhibition of type 1 IFN signaling. ${ }^{10}$ Similarly, susceptibility in C3HebFeJ mice is largely due to a mutation in the Ipr 1 isoform of the If775 gene, which produces type I IFN-driven disease. ${ }^{14}$ The effects of persistent IL1 can be modeled in Nos2-deficient mice as well, since susceptibility in these animals depends on IL1-dependent inflammation. ${ }^{6}$

A common feature of severe TB in both patients and these diverse susceptible mice is the infiltration of granulocytic cells, mostly neutrophils, into the lung. While neutrophil recruitment is critical for protective immunity to many pulmonary pathogens, ${ }^{15,16}$ and certain animal models suggest neutrophils can provide some protection at early stages of mycobacterial infection, ${ }^{17,18}$ multiple lines of evidence in humans and susceptible mice indicate that these cells are pathologic during TB. In humans, neutrophils represent a significant fraction of infected cells observed in sputum, ${ }^{19}$ and biomarkers related to neutrophil function are a strong predictor of disease progression. ${ }^{20,21}$ In mice, depletion of neutrophils from a variety of susceptible lines reduces disease and restores host control

\footnotetext{
${ }^{1}$ Department of Microbiology and Physiological Systems, University of Massachusetts Medical School, Worcester, MA 01655, USA and ${ }^{2}$ Department of Immunology and Microbial Disease, Albany Medical College, Albany, NY 12208, USA

Correspondence: Christopher M. Sassetti (Christopher.sassetti@umassmed.edu)

Pre-publication disclosure of data: Portions of this manuscript were publically presented at the February 2015 Keystone Symposia on Tuberculosis in Santa Fe, New Mexico, at the July 2016 Gordon Research Conference on Microbial Toxins and Pathogenicity in Waterville Valley, NH, and at the January 2017 Keystone Symposia on Tuberculosis in Vancouver, British Columbia, Canada.
}

Received: 8 February 2020 Revised: 10 April 2020 Accepted: 23 April 2020

Published online: 1 June 2020 
of bacterial replication. ${ }^{6,22,23}$ These observations suggest that neutrophil infiltration is a common feature of TB progression promoting both tissue damage and bacterial replication, regardless of underlying causes of susceptibility. While mechanisms behind neutrophil-mediated tissue damage have been described, ${ }^{24}$ it remains unclear why infiltration of these generally antimicrobial cells promotes $M t b$ replication.

Neutrophil infiltration could promote bacterial replication in two fundamentally different ways. First, these cells have known immunoregulatory functions and have been shown to secrete IL10, ${ }^{25}$ a cytokine which inhibits protective T-cell responses in Mycobacterium avium infected mice. ${ }^{26}$ More generally, granulocytes can act as myeloid-derived suppressor cells (MDSCs) capable of inhibiting a protective adaptive immune response via suppression of T-cell function. ${ }^{27-29}$ Alternatively, neutrophils are also phagocytes capable of providing a permissive niche for intracellular $M t b$ replication. ${ }^{30,31}$ Here, we used multiple mouse models of susceptibility to investigate how neutrophil infiltration promotes $M t b$ growth, discovering a subset of lung granulocytic cells that are permissive to $M t b$ infection, express low levels of antimicrobial genes, and exacerbate disease.

\section{RESULTS}

Influx of Ly6G Pos cells into the pulmonary compartment correlates with $M t b$ burden, inflammatory cytokines, and disease severity To determine the connection between neutrophils and Mtb growth, we compared three genetically distinct murine models of disease severity. Wild-type C57BL6 (Bl6 WT) mice are the most resistant to disease, $\mathrm{C} 3 \mathrm{HeBFeJ}$ mice have an intermediate susceptibility associated with type I IFN production, and Nos2 $2^{-1-}$ C57BI6 mice suffer severe IL1-dependent disease. We characterized myeloid cells within these models by cell-surface phenotype (Supplementary Fig. 1), finding that disease severity was reflected by the influx of CD19 ${ }^{\text {Neg }}$ CD $11 b^{\text {High }}$ Ly6G $^{\text {Pos }}$ GR1 $1^{\text {High }}$ Ly6C ${ }^{\text {High }}$ neutrophils into the lung at 28 days post-infection (PI) (Fig. 1a). As reported in other settings, ${ }^{6,32}$ the number of pulmonary neutrophils across the three disease models remained proportional to the number of colony-forming units (CFUs) in the lung (Fig. 1a-graph). Further characterization of myeloid cells revealed that during more severe disease, a subset of Ly6G ${ }^{\text {Mid }}$ granulocytes (CD19 ${ }^{\text {Neg }}$ CD11b ${ }^{\text {High }}$ Ly6G ${ }^{\text {Mid }}$ GR1 ${ }^{\text {Mid }}$ Ly6 $C^{\text {High }}$ ) became apparent (Fig. 1b). The abundance of these cells increased over time, becoming the prominent Ly6G-expressing cell population in Nos $2^{-1-}$ mice at 35 days PI (Fig. 1b). This change in cellular phenotype coincided with an increase in proinflammatory cytokines (Fig. 1c). Collectively, these data suggested a causative link between neutrophil influx, bacterial burden, and hyperinflammatory disease.

Genetic or antibody-mediated depletion of Ly6 $6 \mathrm{G}^{\text {Pos }}$ cells decreases Mtb burden without affecting other immune cells

To quantify the contribution of Ly6G ${ }^{\text {Pos }}$ cell influx to bacterial replication within each setting, we first used antibody-mediated cell depletion to remove Ly6G ${ }^{\text {Pos }}$ cells from infected hosts. Anti-Ly6G (1A8) treatment depleted both $\mathrm{GR} 1^{\text {High }}$ and GR1 ${ }^{\text {Mid }}$ populations from the lungs (Supplementary Fig. 2a). As reported previously, ${ }^{6}$ removal of these cells between days 11 and $23 \mathrm{PI}$ decreased the pulmonary bacterial load (Fig. 2a). The effect of depletion on CFUs correlated with the number of Ly6G ${ }^{\text {Pos }}$ cells, as this treatment had a minimal effect in Bl6 WT mice, but a progressively larger effect in C3HeBFeJ and Nos2 ${ }^{-1-}$ backgrounds. Depletion of Ly6G ${ }^{\text {Pos }}$ cells did not alter the number or phenotype of lymphocytes within the lungs, as we found no difference in the number of total T-cells, total B-cells, FoxP3 ${ }^{\text {Pos }}$ regulatory T-cells (Tregs), or T-bet ${ }^{\text {Pos }}$ Th1-cells between depleted and non-depleted animals (Supplementary Fig. 2b-d). Additionally, depletion did not obviously affect lymphocyte function, as neither the number of IFNY-expressing T-cells nor IL10-expressing
Tregs changed, nor did the amount of IFNy in lung homogenate (Supplementary Fig. 2c, d).

To control for non-specific effects of antibody-mediated depletion and timing-dependent phenotypes, we used a complementary genetic model of neutropenia. C57Bl6 Genista mice specifically lack Ly6G ${ }^{\text {Pos }}$ neutrophils due to a hypomorphic mutation in the gfi1 transcription factor. ${ }^{33}$ We confirmed that these animals do not recruit Ly6G ${ }^{\text {Pos }}$ cells to their lungs upon $M t b$ infection, even upon Nos2 inhibition with aminoguanidine (AG) (Fig. 2b), which otherwise mimics the Nos2 $2^{-1-}$ mutation and increases pulmonary infiltration of Ly6G ${ }^{\text {Pos }}$ cells and CFU load in BI6 WT animals (Supplementary Fig. 3c). As observed during antibody-mediated depletion, genetic abrogation of these cells correlated with reduced bacterial burden, as AG had no effect on bacterial load in Genista mice (Fig. 2b). The lack of Ly6GPos cells in Genista mice also had a minimal impact on the total numbers of macrophages, $\mathrm{CD} 8^{\mathrm{Pos}} \mathrm{T}$-cells, $\mathrm{CD} 4^{\mathrm{Pos}} \mathrm{T}$-cells, and $\mathrm{CD} 19^{\mathrm{Pos}}$ B-cells, even upon AG-treatment to accentuate granulocyte recruitment (Fig. 2c). The modest reduction of other cell types in Genista mice likely reflects the relative reduction in CFUs and concomitant decrease in inflammation, which is consistent with the observed reduced cytokine production (Supplementary Fig. 3a). Thus, we conclude that the disease-attenuating phenotype observed upon Ly6G ${ }^{\text {Pos }}$ cell depletion is directly attributable to loss of Ly6G ${ }^{\text {Pos }}$ granulocytic cells.

Neither granulocyte-derived IL10 nor MDSC activity contribute to disease in $\mathrm{C} 3 \mathrm{HeBFeJ}$ or Nos2 $2^{-1-}$ mice

We next investigated whether IL10 secretion or MDSC activity played a role in promoting disease. Neutrophils from Mtb infected cynomolgus macaques can produce detectable levels of $\mathrm{IL} 10^{25}$, suggesting a possible role for granulocyte-derived IL10 in TB, and Ly6G ${ }^{\text {Pos }}$ cell depletion within our murine models decreased the concentration of IL10 in the lungs of susceptible mouse strains (Supplementary Fig. 3b). However, deletion of the $/ 110$ gene did not alter the number of pulmonary Ly6G ${ }^{\text {Pos }}$ cells in susceptible Nos $2^{-/}$animals, and caused only a minimal decrease in bacterial burden (Fig. 3a).

To specifically probe the antimicrobial impact of granulocytederived IL10 in the lungs, we took advantage of the requirement for C-X-C Motif Chemokine Receptor 2 (CXCR2) in neutrophil recruitment to the pulmonary compartment. ${ }^{6,10,22}$ Even upon AG treatment, we found that, like Genista mice, $C x c r 2^{-1-}$ mice were defective in Ly6G ${ }^{\text {Pos }}$ cell recruitment to the lungs and harbored fewer bacteria than WT controls (Supplementary Fig. 3c). Using this model, we constructed $50: 501110^{-1-}: \mathrm{CxCr}^{-/-}$bone-marrow chimeric mice where CXCR2 expression within the granulocyte compartment was restricted to IL10-deficient cells, and thus only those IL10-deficient Ly6G ${ }^{\text {Pos }}$ granulocytes could traffic to the lungs (Fig. 3b, c). Upon Mtb infection, we observed no difference in CFU load between animals with IL10-deficient or IL10-sufficent Ly6G Pos cells (Fig. 3d), even when granulocyte recruitment was augmented by AG treatment. Together, these data indicated that Ly6G ${ }^{\text {Pos }}$ cells may contribute to total IL10 levels in the lungs, but their IL10 production does not play a significant role in controlling antimicrobial immunity in this model.

We next isolated myeloid cell subsets from $M t b$-infected mouse lungs to assess their MDSC functionality. These experiments specifically tested the hypothesis that Ly6G ${ }^{\text {Mid }}$ granulocytes, which resemble cells associated with MDSC activity in 129SvPas mice, ${ }^{27}$ exacerbate disease by suppressing T-cell function in our models. We observed that Ly6G ${ }^{\text {Mid }}$ granulocytes from susceptible C3HebFeJ and Nos2 $2^{-1-}$ mice express inconsistent levels of MDSC markers, with overall lower expression of CD47, CXCR4, CD49d, c-kit, and CD115, yet increased levels of CD124 compared to Ly6G ${ }^{\text {High }}$ neutrophils (Supplementary Fig. 3d, e). To determine if these cells express relevant regulatory activity, we performed T-cell suppression assays using isolated Ly6G ${ }^{\text {High }}$ neutrophils or Ly6G ${ }^{\text {Mid }}$ granulocytes (Fig. 3e) from Bl6 WT, 129SvPas, C3HebFeJ, 
a

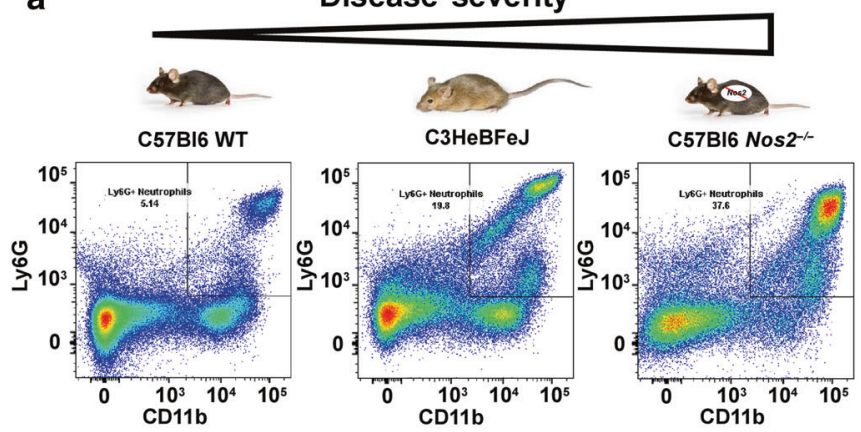

\section{Disease severity}

b
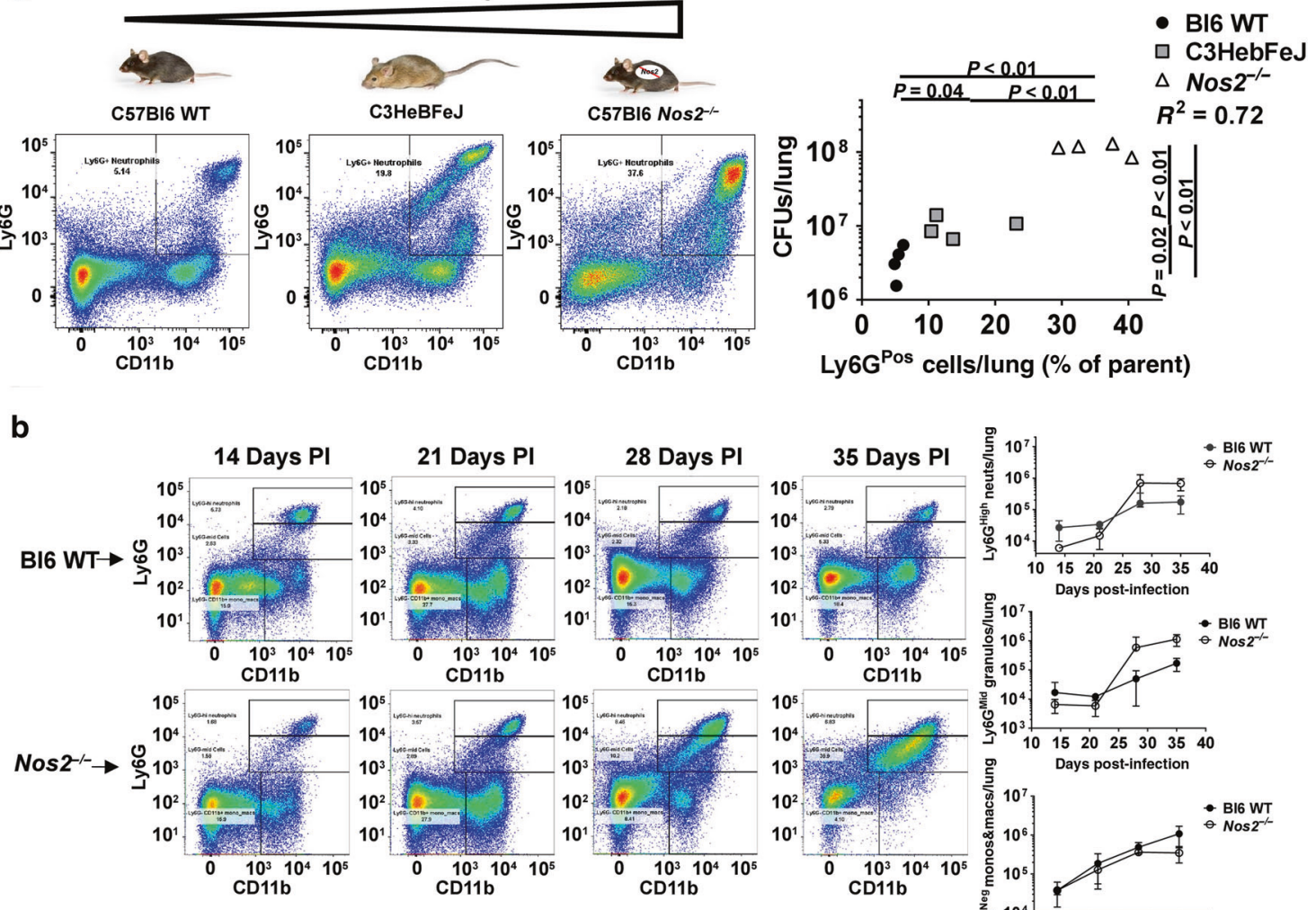

Ly6G ${ }^{\text {Pos }}$ cells/lung (\% of parent)

c

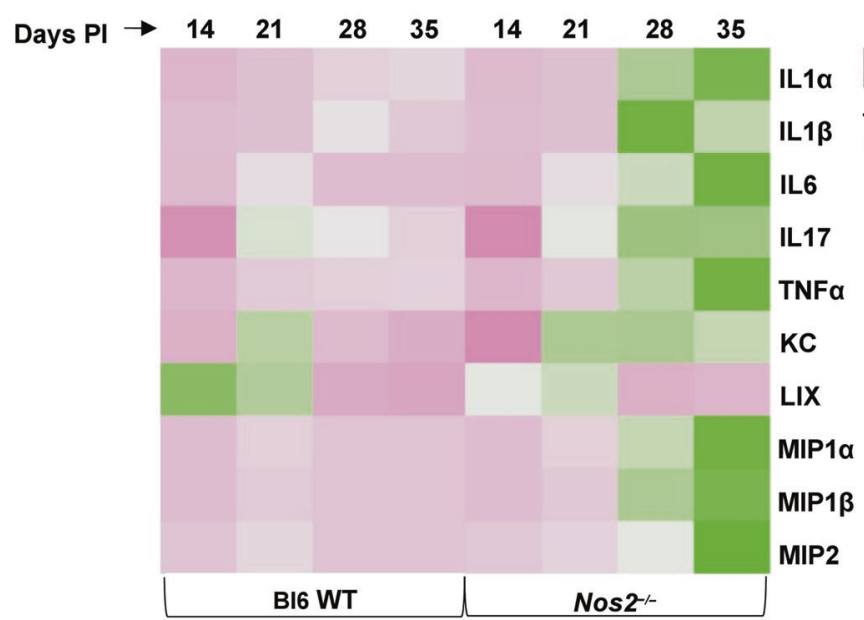

Fig. 1 Influx of Ly6G ${ }^{\text {Pos }}$ cells into the pulmonary compartment correlates with Mtb burden, inflammatory cytokines, and disease severity. a Mouse models of increasingly severe disease (left to right), with representative scatterplots of CD11 b ${ }^{\text {High }}$ Ly6G ${ }^{\text {Pos }}$ pulmonary cells at 28 days $\mathrm{PI}$, and quantified (far right) as percent Ly6G ${ }^{\text {pos }}$ cells by recovered CFUs in the lung of each individual mouse. b Representative scatterplots (left) and quantification (right) of CD11 $b^{\text {High }}$ Ly6G ${ }^{\text {High }}$ neutrophils (top right), CD11 $b^{\text {High }}$ Ly6G ${ }^{\text {Mid }}$ granulocytes (middle right), and CD11 $\mathrm{b}^{\text {High }}$ Ly6G ${ }^{\mathrm{Neg}}$ monocytes/macrophages (bottom right) in Bl6 WT or Nos $2^{-/-}$mice at the indicated time points PI. $N=4$ mice per group and error bars represent standard deviation between biological replicates within each group. c Multiplex analysis of the indicated cytokines and chemokines

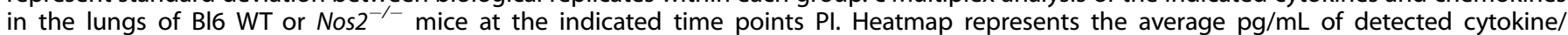
chemokine from 4 mice per group scaled by row.

or Nos $2^{-1-}$ mice. Consistent with previous findings, ${ }^{27}$ we observed significant suppression of IFNy production from activated T-cells co-cultured with either Ly6G ${ }^{\text {High }}$ or Ly6G ${ }^{\text {Mid }}$ cells from 129SvPas mice (Fig. 3f). In contrast, we observed no suppression from Bl6 WT, C3HebFeJ, or Nos2 $2^{-1-}$ cells (Fig. 3f). Thus, while functionally different subsets of granulocytes may infiltrate the lungs based on the root cause of susceptibility, MDSC-based T-cell suppression does not explain the disease-promoting effect of $L y 6 G^{\text {Pos }}$ cells in C3HebFeJ and $\mathrm{Nos}^{-1-}$ mice.

Mtb preferentially associates with Ly6G ${ }^{\text {Pos }}$ cells during loss of immune containment

Data to this point indicated that $L y 6 G^{\text {Pos }}$ cells promote bacterial replication without altering common markers of protective adaptive 

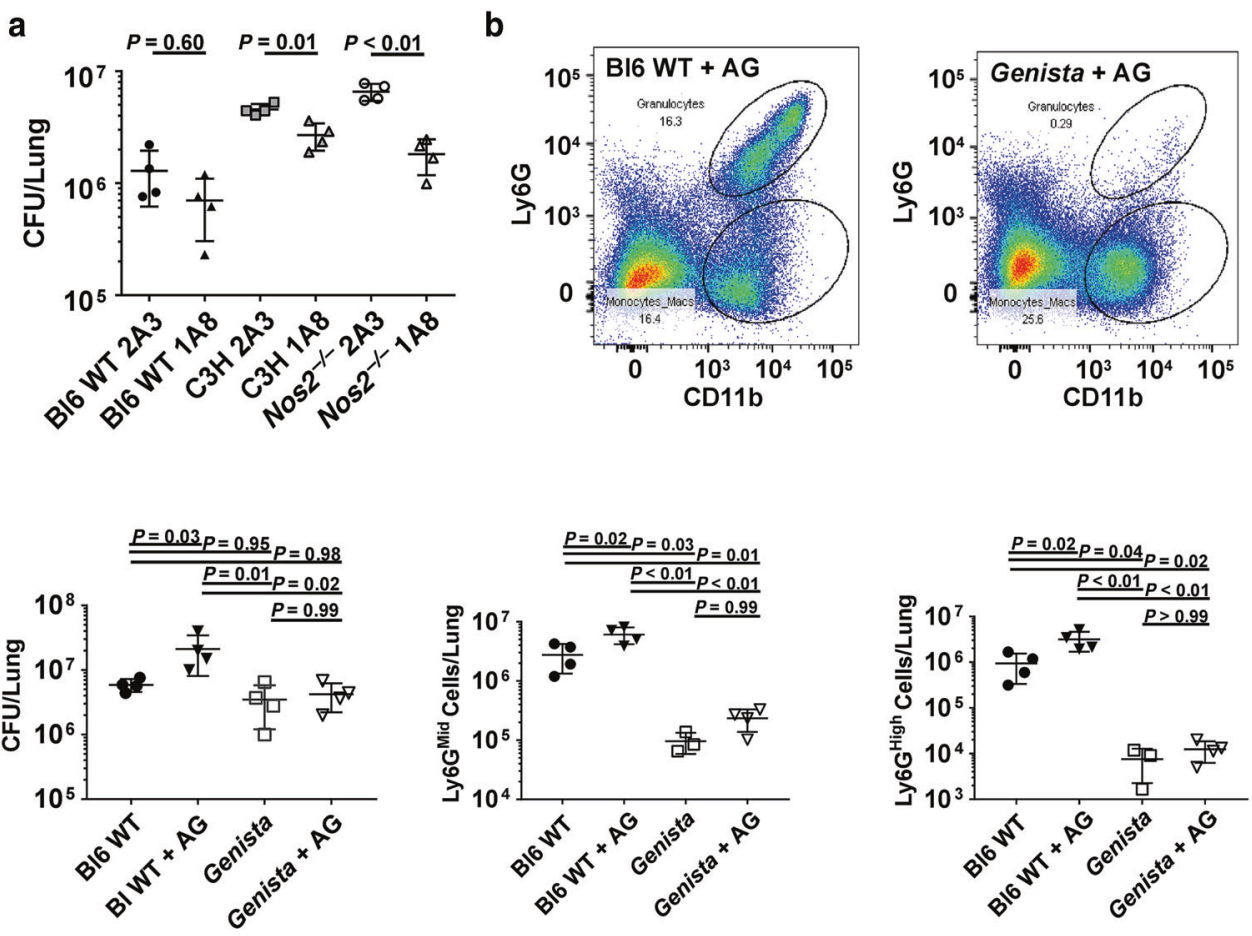

C

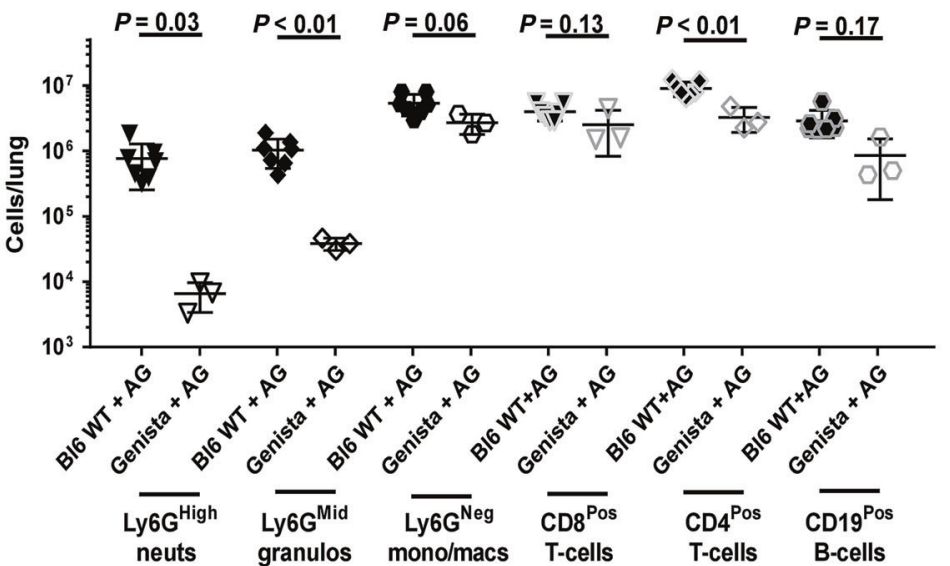

Fig. 2 Genetic or antibody-mediated depletion of Ly6G ${ }^{\text {Pos }}$ cells decreases Mtb burden without affecting other immune cells. a CFUs per lung at 25 days PI within Bl6 WT, C3HebFeJ, or Nos $2^{-1-}$ mice treated with anti-Ly6g depleting antibody (1A8) or isotype control (2A3). b Representative scatterplots of lung cells from infected BI6 WT (top mid) or Genista (top right) mice treated with aminoguanidine (AG), with quantified CFUs (bottom left), Ly6G ${ }^{\text {Mid }}$ cells (bottom mid), or Ly6G ${ }^{\text {High }}$ cells (bottom right) per lung at 56 days PI with or without AG treatment. c Quantification of the indicated immune cells in the lungs of AG-treated BI6 WT mice or AG-treated Genista mice at 56 days PI. N=3-7 mice per group and error bars represent standard deviation between biological replicates within each group. Statistical values based on unpaired Student's T-test or one-way ANOVA with Tukey's Multiple Comparison analysis.

immunity. As a result, we hypothesized that the increased number of granulocytes in the lungs of susceptible animals instead confers a more favorable niche for $M t b$ replication. Consistent with this model, we previously found that the number of infected CD11b $\mathrm{b}^{\text {High }}$ Ly6G ${ }^{\text {Pos }}$ granulocytic cells exceeded infected monocytes/macrophages in Nos $2^{-1-}$ animals. $^{6}$ To further test this hypothesis, we quantified the number of bacteria in various niches, including myeloid cell subsets and extracellular sites, representing significant replicative sites in necrotic lesions.

To estimate relative bacterial burden within intra- and extracellular niches, we developed an assay utilizing differential centrifugation and a cell-impermeable antibiotic to calculate extracellular, cell-associated, or intracellular Mtb fractions within the lungs (Supplementary Fig. 4a), concentrating on the extremes of susceptibility: Bl6 WT and Nos2 $2^{-1-}$. We observed no difference in the number of intracellular bacteria compared to total cellassociated bacteria at 14,21 , or 28 days $\mathrm{Pl}$, indicating that the vast majority of cell-associated Mtb were internalized (Supplementary Fig. 4b). While we did observe a measurable population of extracellular bacteria, the proportion recovered from intra- and extracellular sites remained relatively constant over time (Fig. $4 a$ and Supplementary Fig. 4c). Thus, increased extracellular replication is unlikely to explain the excessive bacterial burden observed in $\mathrm{Nos}^{-/-}$mice.

To assess bacterial load within distinct myeloid subsets, we developed a flow cytometry assay to quantify the number of bacteria per cell using a fluorescent msfYFP-expressing strain of $M t b .^{6}$ The calculated YFP signal per cell (YFP Units) correlated with 
a

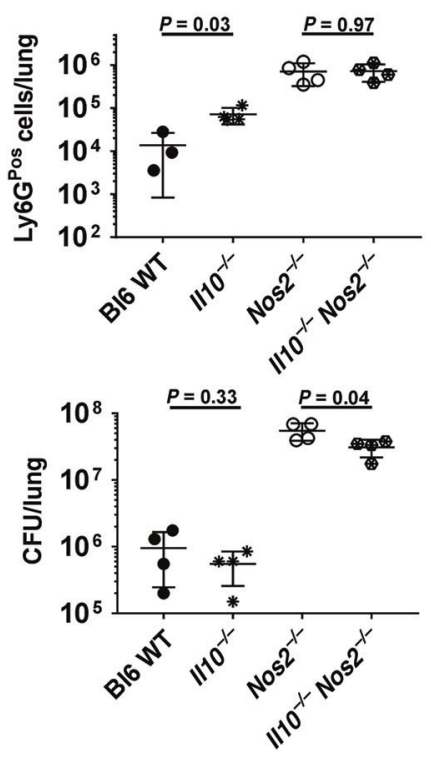

C

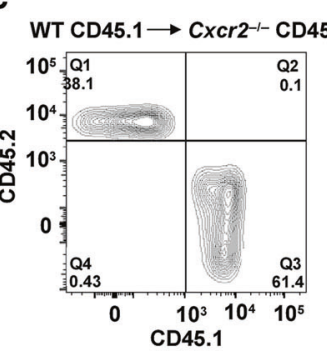

e
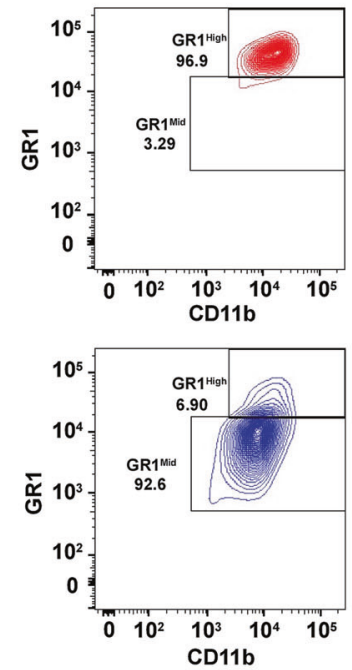

b

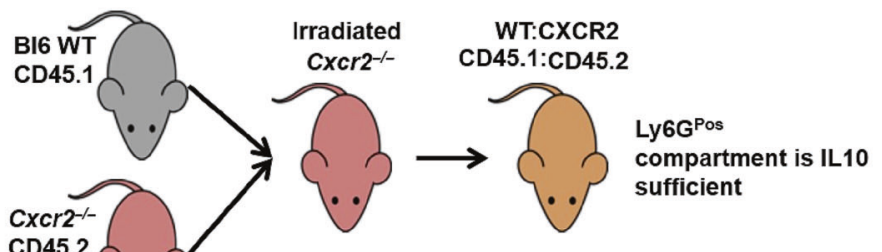

50:50 bone-marrow

reconstitution into

Cxcr2 ${ }^{-/-}$mice

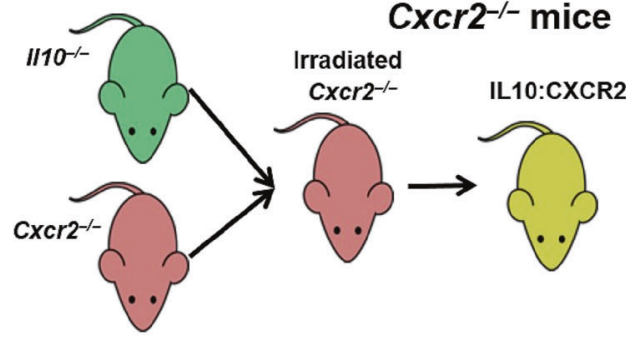

Ly6GPos compartment is

IL10 deficient

Fig. 3 Neither granulocyte-derived IL10 nor MDSC activity contribute to disease in C3HeBFeJ or Nos2 ${ }^{-1-}$ mice. a Number of Ly6G ${ }^{\text {Pos }}$ cells (top) or CFUs (bottom) in the lungs of the indicated mouse strains at 28 days PI. b Schematic diagram of mouse chimerism to produce 50:50 WT:CxCr2 ${ }^{-/-}$mice (where the Ly6G ${ }^{\text {Pos }}$ compartment is IL10 sufficient) or $50: 501 / 10^{-/-}: C x C r 2^{-/-}$mice (where the Ly6G ${ }^{\text {Pos }}$ compartment is IL10

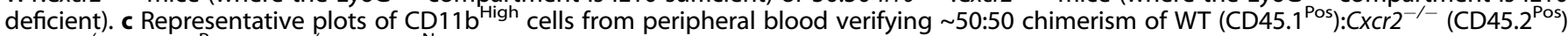
or $1110^{-1-}\left(C X C R 2^{\mathrm{Pos}}\right): \mathrm{CXCr}^{-1-}\left(\mathrm{CXCR2} 2^{\mathrm{Neg}}\right)$. d CFUs recovered from the lungs or spleen of AG-treated chimeric or control mice at 28 days PI. e Representative plots of CD11 $\mathrm{b}^{\mathrm{High}}$ Ly6G ${ }^{\text {Pos }}$ cells recovered from infected Nos2 ${ }^{-1-}$ mice, using the Miltenyi MDSC isolation kit, verifying the isolation of subpopulations via GR1 expression. $\mathbf{f}$ Total IFN $\gamma$ production by activated T-cells co-cultured at a 1:1 ratio over $24 \mathrm{~h}$ with Ly6G ${ }^{\text {High }}$ or Ly $6 \mathrm{G}^{\mathrm{Mid}}$ cells of the indicated genotype recovered at 28 days $\mathrm{PI} . N=4$ mice per group and error bars represent standard deviation between these biological replicates. Statistical values based on one-way ANOVA with Tukey's Multiple Comparison analysis. 
a

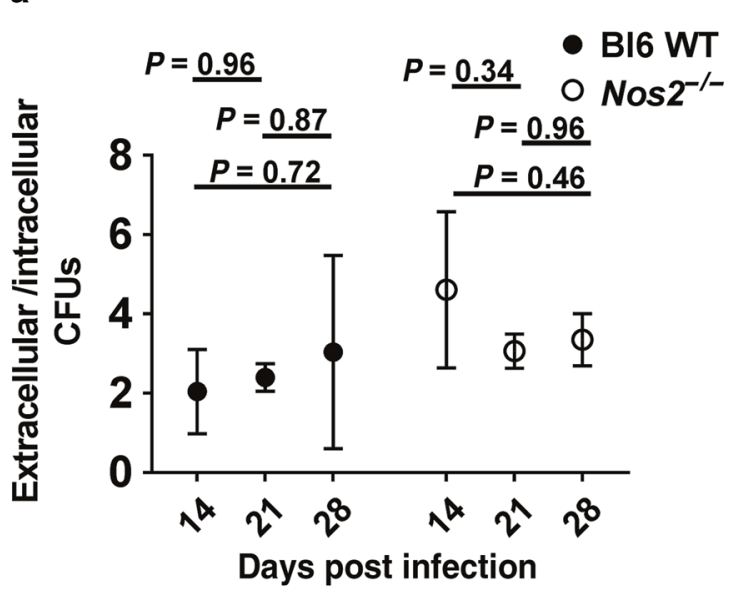

b

- BI6 WT CFUs

BI6 WT YFP units

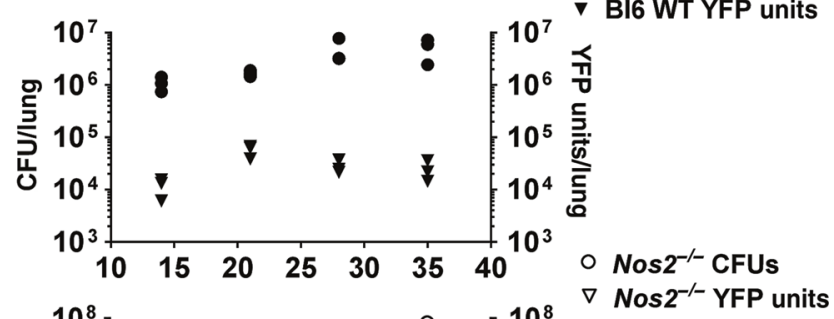

c
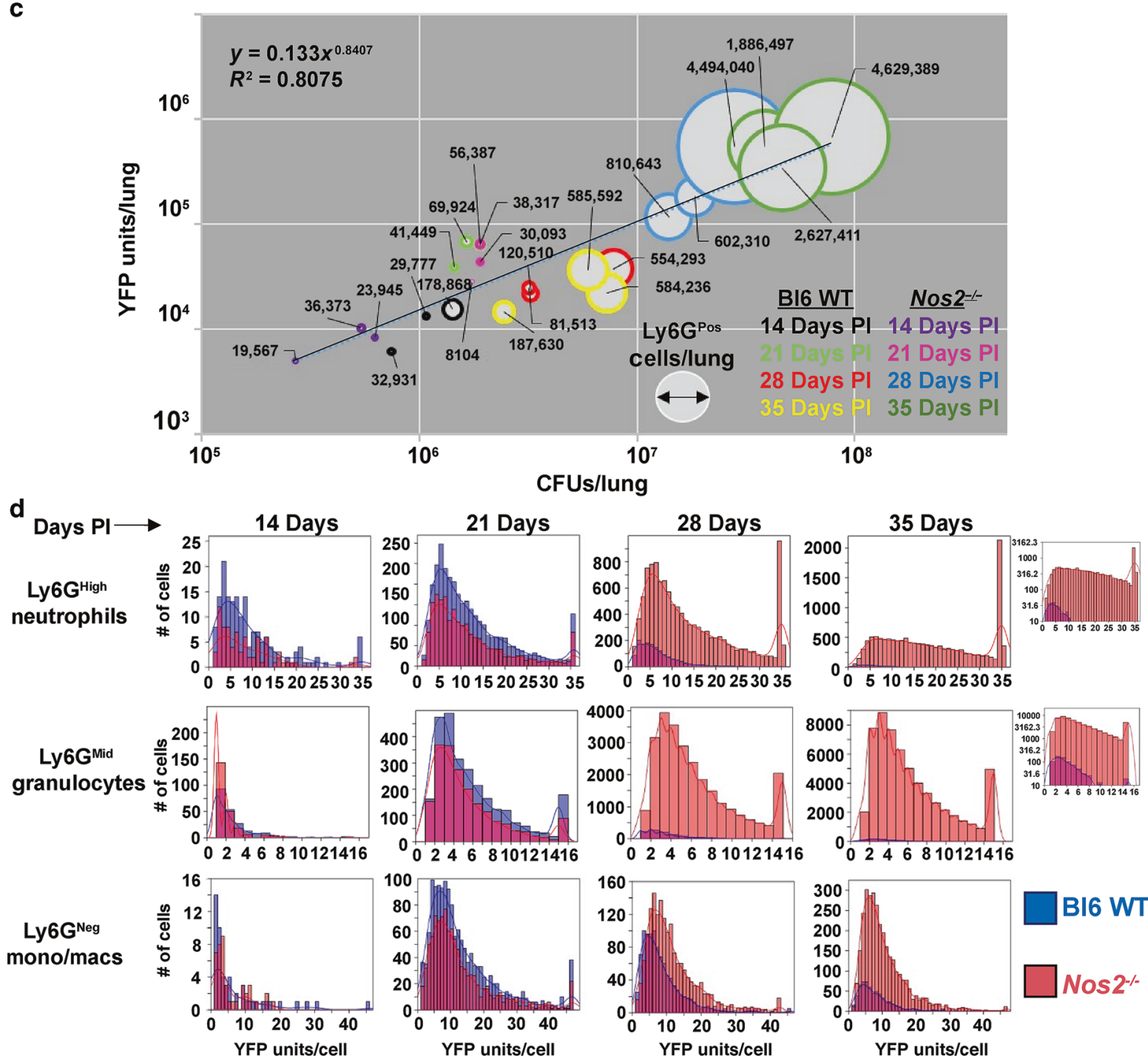

BI6 WT

Nos2 -1

recovered CFUs from lungs over time (Fig. 4b), and thus served as a proxy for measuring bacterial load within individual cells. Using this assay, we found a strong correlation between total bacterial numbers, duration of infection, and abundance of Ly6G ${ }^{\text {Pos }}$ cells in the lungs of B6 WT and Nos2 ${ }^{-1-}$ mice (Fig. 4c).
When we individually assessed the distribution of bacteria per cell within different myeloid classes, we found a clear difference between Ly6G ${ }^{\text {Pos }}$ and Ly6G ${ }^{\text {Neg }}$ populations as disease progressed (Fig. 4d). During the first 21 days of infection, Nos2 had little effect on the cellular composition of the lung (Fig. 1b), and we observed 
Fig. 4 Mtb preferentially associates with Ly6G ${ }^{\text {Pos }}$ cells during loss of immune containment. a Ratio of extracellular/intracellular CFUs recovered from the lungs of msfYFP-Mtb infected BI6 WT or Nos2 $2^{-/-}$mice at the indicated time points. Error bars represent standard deviation between mice within each group. Statistical values based on one-way ANOVA with Tukey's Multiple Comparison analysis. b CFUs (circles, left Y-axis) or normalized YFP Units (triangles, right Y-axis) in the lungs of msfYFP-Mtb infected Bl6 WT (top) or Nos2 ${ }^{--}$(bottom) mice at the indicated time points. Each data point represents 1 mouse. c YFP units (Y-Axis) as a function of CFUs (X-axis) and Ly6G ${ }^{\text {Pos }}$ cell number (width of circles with numerical label) in the lungs of infected Bl6 WT or Nos2 $2^{-1-}$ mice. Time points are indicated by coloring. "R2" indicates the correlation coefficient for the fitted line. d Number of YFP Units per cell in msfYFP-Mtb associated CD11 b ${ }^{\text {High }}$ Ly6G $^{\text {High }}, C^{2}$. $11 b^{\text {High }}$ Ly6G $^{\text {Mid }}$, or CD11 $b^{\text {High }}$ Ly6G $^{\text {Neg }}$ cells from BI6 WT (blue bars) or Nos $2^{-1-}$ (pink bars) mice at the indicated timepoints. Inset: YFP Units per cell within Ly6G ${ }^{\text {High }}$ or Ly6G ${ }^{\text {Mid }}$ compartments at 35 days PI, graphed on a log Y-axis for ease of visualization. $N=3$ mice per group per time point, or 3 mice per group per bar graph.

only modest differences in the distribution of bacteria among the three cell classes (Ly6G ${ }^{\text {High }}$ neutrophils, Ly6G ${ }^{\text {Mid }}$ granulocytes, and Ly6G ${ }^{\mathrm{Neg}}$ monocytes/macrophages) between Bl6 WT or Nos2 ${ }^{-1-}$ mice (Fig. 4d). However, the impact of Nos2 became apparent after 28 days. At this point, the total bacterial burden in Bl6 WT mice had stabilized as a result of the adaptive response (Fig. 4b), and this control was reflected in monocytes/macrophages where both the number of infected cells and bacteria per cell remained relatively consistent across both genotypes (Fig. $4 d$ and Table 1). While the number of infected granulocytes, as well as the number of bacteria per granulocyte, decreased between 21 and 28 days PI in Bl6 WT mice, the opposite effect occurred in granulocytes within Nos2 $2^{-/-}$ mice. In the Nos2 $2^{-/-}$background, the number of infected Ly6G ${ }^{\text {High }}$ and Ly6G ${ }^{\text {Mid }}$ cells increased by 4.7 - or 12.3 -fold, respectively. The average number of bacteria per Ly6G ${ }^{\text {Pos }}$ granulocyte remained relatively constant, increasing by $\sim 20 \%$ (Table 1-highlighted with bold). These trends continued as disease progressed in $N o s 2^{-/-}$ mice. By 35 days PI, the number of infected Ly6G ${ }^{\text {Neg }}$ monocytes/ macrophages had increased in Nos $2^{-1-}$ mice, but we observed the vast majority of the bacterial burden within Ly6G Pos cells, most of which displayed the Ly6G ${ }^{\text {Mid }}$ phenotype (Fig. 4d). These data are consistent with a model in which the protective adaptive response preferentially restricts bacterial growth in $L y 6 G^{\text {Neg }}$ monocytes/ macrophages verses Ly6G ${ }^{\text {Pos }}$ granulocytic cells, and recruitment of these more permissive granulocytes promotes infection.

Ly6G ${ }^{\text {Mid }}$ granulocytes are retained in the lungs at least as long as monocytes and macrophages

$M t b$ is canonically thought to replicate in relatively long-lived myeloid cells. The lifespan of myeloid cell populations can be highly variable, dependent on activation status, sub-lineage, tissue residence, and disease-specific regulation. ${ }^{34}$ Within Mtb-infected lungs, the lifespan of monocytic phagocytes, which are canonically considered a niche for Mtb, relative to granulocytic cells has never been assessed. To address this, we developed an assay to calculate the half-life of pulmonary myeloid cells in vivo (Fig. 5a). One hour after the instillation of a nonspecific fluorescent cell stain via intratracheal aspiration, approximately $60 \%$ of all viable lung cells stained positive by flow cytometry (Fig. 5a and Supplementary Fig. 5a). Analysis of spleens verified that the dye does not escape the pulmonary compartment (Supplementary Fig. 5b), and analysis of long-lived pulmonary CD19 ${ }^{\text {Pos }} \mathrm{B}$-cells indicated stain retention up to at least $48 \mathrm{~h}$ post-treatment (Supplementary Fig. $5 \mathrm{c}$ ). Based on the average number of stain-positive cells at 1-h post-treatment versus 48-h post-treatment, the calculated half-life of pulmonary bulk Ly6G ${ }^{\text {Neg }}$ monocytes/macrophages, Ly6G Mid granulocytes, and Ly6G ${ }^{\text {High }}$ neutrophils from either Bl6 WT or Nos $2^{--1}$ mice at 28 days PI was $22 \pm 8 \mathrm{~h}, 40 \pm 10 \mathrm{~h}$, and $12 \pm 2 \mathrm{~h}$, respectively (Fig. 5b). Using the msfYFP-Mtb strain, we found the half-life of infected and uninfected cells was similar within cell types, and host genotype had a relatively modest effect (Fig. 5c), suggesting that differences between cellular life spans are intrinsic to the cell type and not strongly influenced by inflammatory state of the organ or direct effects of the bacterium. These collective data indicate

Table 1. Bacterial burden within myeloid cell populations from BI6 WT or Nos2 $2^{-/-}$mice.

21 Days PI 28 Days PI \% Change

Bl6 WT Ly6G high neutrophils

Average bacteria per cell

Median of the distribution

Mode of the distribution

Range of the distribution

\section{2}

9

5

Average \# infected cells per lung 1100

B16 WT Ly6G mid granulocytes

Average bacteria per cell

Median of the distribution

Mode of the distribution

Range of the distribution

Average \# infected cells per lung 900

Bl6 WT Ly6G Neg Mono/Macs

Average bacteria per cell

Median of the distribution

Mode of the distribution

Range of the distribution

Average \# infected cells per lung 490

Nos2 ${ }^{-1-}$ Ly6G high neutrophils

\section{Average bacteria per cell}

Median of the distribution

Mode of the distribution

Range of the distribution

Average \# infected Cells per Lung 700

\section{Nos2 ${ }^{-/}$Ly6G Mid granulocytes}

$\begin{array}{llll}\text { Average bacteria per cell } & \mathbf{5} & \mathbf{6} & \mathbf{2 0} \\ \text { Median of the distribution } & 4 & 5 & 25 \\ \text { Mode of the distribution } & 2 & 3 & 50 \\ \text { Range of the distribution } & 1-15 & 1-15 & 0 \\ \begin{array}{l}\text { Average \# infected Cells per Lung } \\ \text { Nos2 }{ }^{-/-} \text {Ly6G Neg Mono/Macs }\end{array} & 660 & 8800 & 1233 \\ \begin{array}{l}\text { Average bacteria per Cell } \\ \text { Median of the distribution }\end{array} & 13 & 12 & -8 \\ \text { Mode of the distribution } & 8 & 9 & -10 \\ \text { Range of the distribution } & 1-47 & 1-43 & \sim \\ \text { Average \# infected cells per Lung } & 345 & 560 & 62\end{array}$

The average number of infected cells per lung, with the mean, median, range, and mode of the distribution of YFP Units per cell represented for the indicated cell types and genotypes at 21 days or 28 days PI. \% change represents the percent difference from 21 to 28 days $\mathrm{PI}$ of the indicated metric. $N=3$ mice per genotype per time point. 

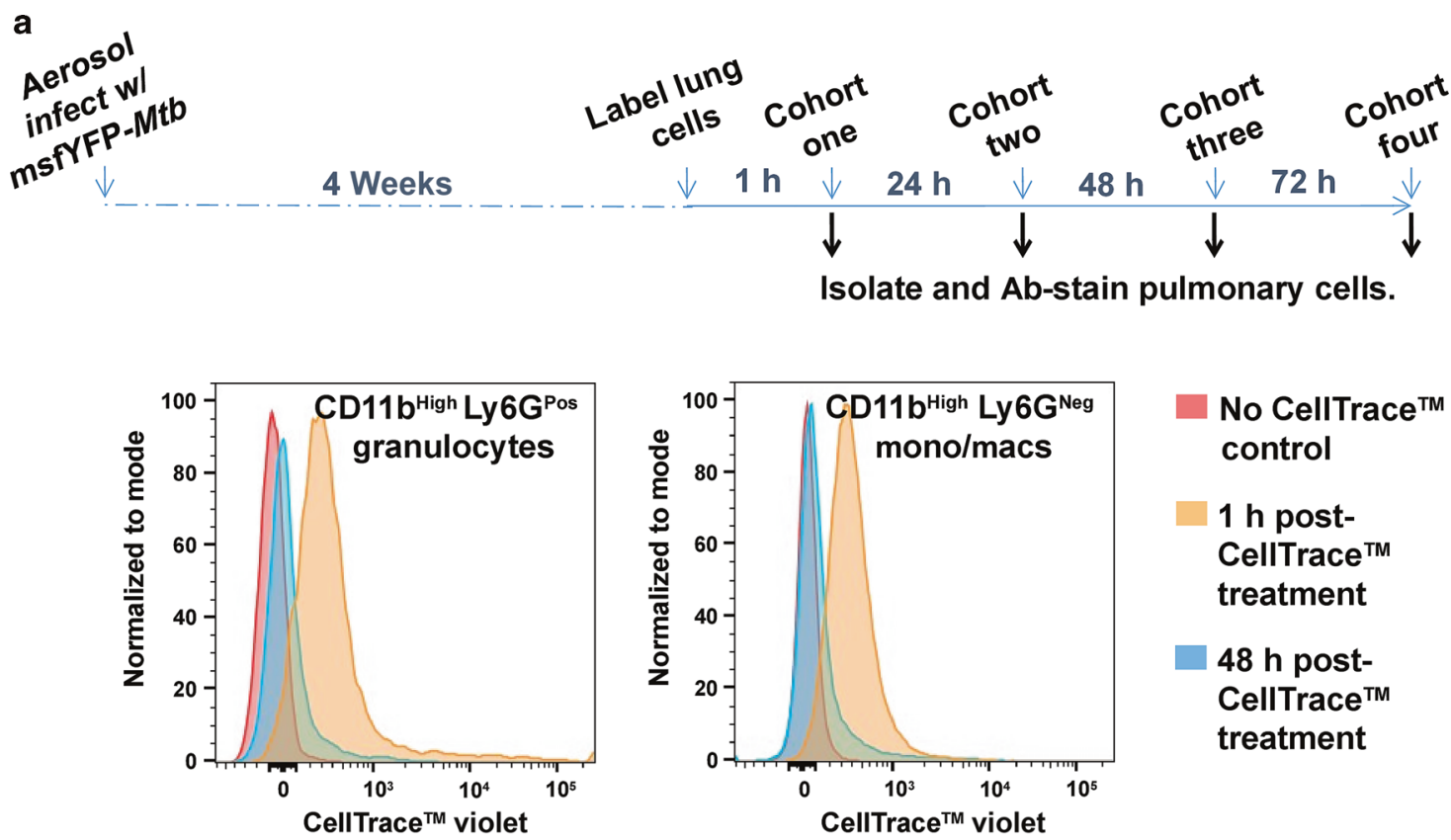

No CellTrace ${ }^{\mathrm{TM}}$ control

$1 \mathrm{~h}$ postCellTrace $^{\mathrm{TM}}$ treatment

$48 \mathrm{~h}$ postCellTrace ${ }^{\mathrm{TM}}$ treatment

b

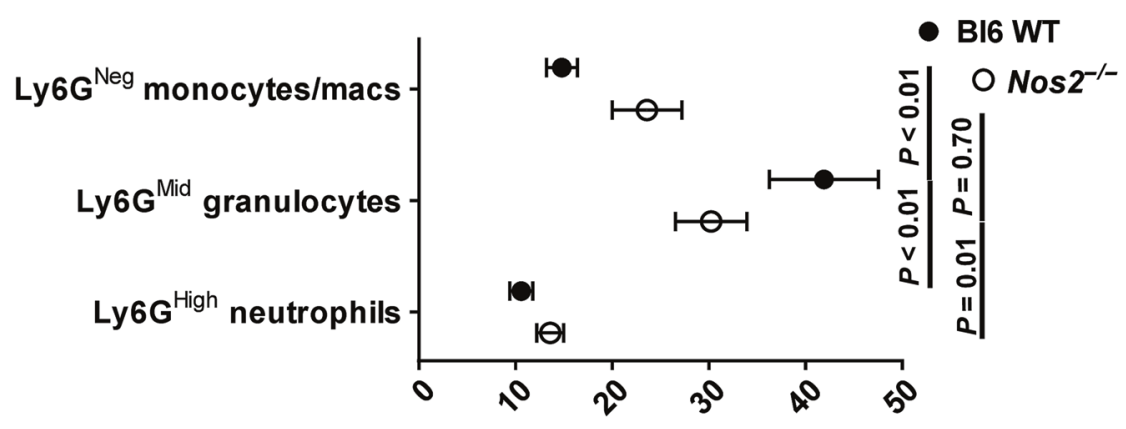

Avg. half-life (h)

C

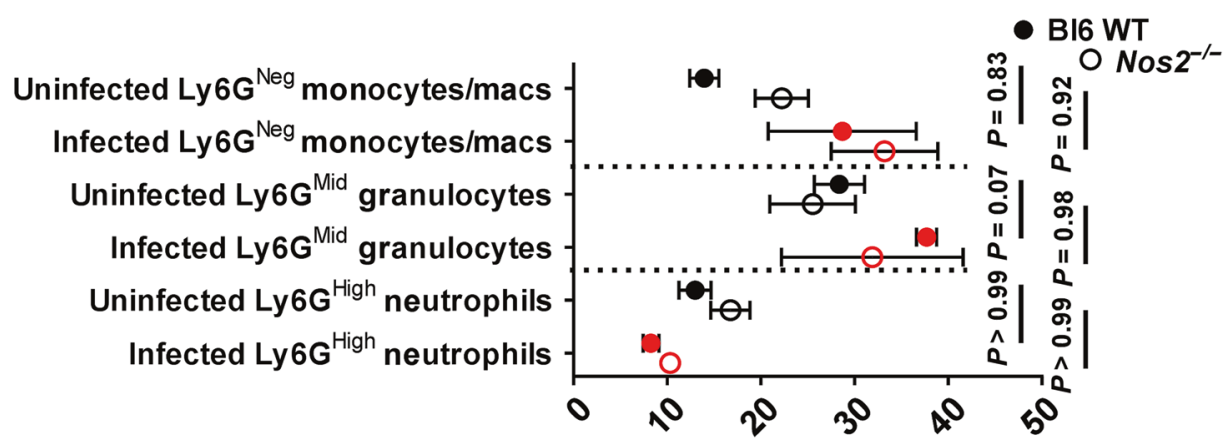

Avg. half-life (h)

Fig. 5 Ly6G ${ }^{\text {Mid }}$ granulocytes are retained in the lungs at least as long as monocytes/macrophages. a Schematic of in vivo pulmonary cell half-life assay (top) and representative histogram overlays (bottom) of Bl6 WT pulmonary CD11 ${ }^{\text {High }}$ Ly6G $^{\text {Pos }}$ cells or CD11 $^{\text {High }}$ Ly6G $\mathrm{G}^{\text {Neg }}$ monocytes/macs left untreated (red) or stained with CellTrace ${ }^{T M}$ Violet via tracheal aspiration and measured at $1 \mathrm{~h} \mathrm{(gold)} \mathrm{or} 48 \mathrm{~h}$ (blue) postlabeling. b Mean half-life of total or c infected YFP Pos (red) vs uninfected YFP ${ }^{\text {Neg }}$ (black) Ly6G ${ }^{\text {Neg }}$ monocytes/macrophages, Ly6G ${ }^{\text {Mid }}$ granulocytes, or Ly6G ${ }^{\text {High }}$ neutrophils in the lungs of BI6 WT or Nos2 ${ }^{-1-}$ mice. Error bars represent standard deviation between half-life values calculated from at least 3 separate pairs of biological replicates (mice). $N=3$ mice per genotype per time-point post-treatment. Statistical values based on two-way ANOVA with Tukey's Multiple Comparison analysis.

that certain Ly6G Pos populations, specifically Ly6G ${ }^{\text {Mid }}$ cells, have a lifespan exceeding that of canonical monocyte/macrophage compartments for intracellular $M t b$ replication. These findings are thus consistent with a model in which Mtb utilizes Ly6G ${ }^{\text {Pos }}$ cells as a replicative niche for intracellular growth.
Gene expression profiling of Ly6G ${ }^{\text {Mid }}$ granulocytes indicates decreased antimicrobial function and proinflammatory effects To further characterize the long-lived Ly6G ${ }^{\text {Mid }}$ cell subset, we performed RNAseq analysis. In steady-state, neutrophils fully differentiate in the bone-marrow before circulating in the blood, ${ }^{35}$ 
and much of our understanding of neutrophil function comes from studying this mature cell type. Due to the well-documented heterogeneity within granulocyte populations, ${ }^{35,36}$ we first contrasted gene expression differences between pulmonary Ly6G Mid granulocytes from infected Bl6 WT mice and canonical Ly6GHigh peripheral blood neutrophils recovered from naïve BI6 WT mice. Gene ontology analysis revealed significant differential expression of immune process genes between the two data sets. Of the top 30 most differentially expressed genes, Ly6G ${ }^{\text {Mid }}$ granulocytes expressed lower levels of genes with antimicrobial functions such as those regulating phagosome activity (Neutrophil Granule Protein $\left.(\mathrm{Ngp})^{37}\right)$, sequestering iron (Lipocalin $(\mathrm{LCn} 2)^{38}$ and Lactoferrin $\left.(L t f)^{39}\right)$, direct killing of the bacterium (Cathelicidin Antimicrobial Protein $\left.(\mathrm{Camp})^{40}\right)$, or regulating anti-mycobacterial properties (CCAAT Enhancer Binding Protein Epsilon $(C E B P)^{7,41}$ ) (Fig. $6 a$ and Supplementary Table 1). To better understand the physiological basis of these differences, we searched for potential upstream regulators that control these genes. This analysis predicted altered activity of Mrtfb, which encodes the MRTF-SRF transcriptional coactivator of serum response factor (Fig. 6b). MRTF-SRF has been identified as a regulator of hematopoiesis and neutrophil migration, ${ }^{42}$ and our data suggest that Mrtfb may also coordinate granulocyte responses during $M t b$ infection.

To further define this granulocyte subpopulation relative to activated Ly6G ${ }^{\text {High }}$ pulmonary neutrophils present during Mtb infection, we performed analyses as described above comparing Ly6G ${ }^{\text {Mid }}$ granulocytes to Ly6G ${ }^{\text {High }}$ neutrophils recovered from the lungs of Mtb-infected Nos $2^{-1-}$ mice, where both subsets are prevalent. Many of the top 30 most differentially expressed genes were chemotactic factors, such as $\mathrm{Ccr} 2$ and $\mathrm{Ccl} 4$, which encode $\mathrm{C}-\mathrm{C}$ Motif Chemokine Receptor 2 and C-C Motif Chemokine Ligand 4, respectively, and inflammatory regulators such as Ltb, Csf1, and Igfir, which encode Lymphotoxin Beta, Macrophage ColonyStimulating Factor 1, and Insulin-like Growth Factor 1 Receptor, respectively (Fig. 7a and Supplementary Table 2). Consistent with our experimental evidence demonstrating that lung granulocytes contribute to inflammatory cytokine production (Supplementary Fig. 3a), pathway analysis elucidating potential regulatory networks revealed that $\mathrm{Ly}_{6 \mathrm{G}}{ }^{\mathrm{Mid}}$ granulocytes exhibit an expression pattern predicted to promote inflammation (Fig. 7b). Taken together, our data suggest that Ly6G ${ }^{\text {Mid }}$ granulocytes represent a granulocyte subclass that is less antimicrobial and more inflammatory than Ly6G ${ }^{\text {High }}$ neutrophils.

\section{DISCUSSION}

While granulocyte influx is a hallmark of pulmonary $\mathrm{TB}^{19}$ the disease-impacting roles of these cells are still being elucidated. Ly6G Pos granulocytic cells are now recognized as a heterogeneous population capable of differential signaling, regulatory, and antimicrobial functions. ${ }^{27,35,36}$ In this work, we investigated the phenotype and role of these cells in Mtb-susceptible mouse strains. Our data indicate that Ly6G Pos cells directly promote bacterial replication and can serve as a primary niche for Mtb in susceptible hosts. Several lines of evidence, summarized below, support a model in which the recruitment of granulocytes, a Ly6G ${ }^{\text {Mid }}$ subpopulation in particular, provides a favorable site for Mtb replication.

Granulocyte depletion ameliorates disease in multiple models of TB susceptibility without perturbing other aspects of the immune response. While the anti-Ly6G cell-depleting antibody $1 \mathrm{~A} 8$ is a common tool for the study of these cells in mice, ${ }^{6,23}$ the mechanism(s) by which depletion occurs remain(s) obscure, ${ }^{43}$ and potential off-target effects can cloud experimental conclusions. The Genista mouse model offers a genetic alternative to antibodymediated depletion that is maintained throughout infection yet still specific to Ly6G ${ }^{\text {Pos }}$ cells. ${ }^{33}$ We utilized both models to verify that removal of Ly6G ${ }^{\text {Pos }}$ cells from susceptible mice reduced Mtb growth without altering adaptive immunity. These data indicate that Ly6G ${ }^{\text {Pos }}$ cells directly promote Mtb growth.

Known immunomodulatory functions of neutrophils did not influence TB progression in the models used in this study. Previous reports have found granulocytic MDSCs can contribute to disease through suppression of T-cell antimicrobial activity in 129SvPas mice. ${ }^{27,28}$ While we also detected suppressive activity in 129SvPas granulocytes, this effect was not generalizable to Bl6 WT, C3HeBFeJ, or Nos2 $2^{-1-}$ mice. This difference was particularly surprising for $129 \mathrm{SvPas}$ and $\mathrm{C} 3 \mathrm{HeBFeJ}$ mice, as susceptibility in both settings is due to a type I IFN response. We conclude that suppressive functionality depends on genetic background, but is not required for granulocytes to promote mycobacterial replication.

The apparently direct effect of granulocytes on bacterial burden and lack of obvious immunoregulatory activity suggested that granulocyte recruitment instead produced a more favorable replicative niche. Consistent with this hypothesis, the adaptive immune response in the Nos $2^{-1-}$ background was capable of at least transiently controlling bacterial burden in monocytes/ macrophages, but not in Ly6G Pos cells (Fig. 4d). As disease progressed, the Ly6G ${ }^{\text {Mid }}$ subpopulation became the most abundant infected granulocyte, suggesting a particularly important role for these cells once immune containment is lost. In other contexts, Ly6G ${ }^{\text {Mid }}$ granulocytes have been described as immature neutrophils. ${ }^{44}$ Using known markers of maturation, such as CD49d, c-kit, CD35, CD64, and IFNYR (Supplementary Fig. 3e and data not shown), ${ }^{45-47}$ we were unable to define these cells as specific neutrophil precursors. Similarly, we previously observed a heterogeneous cytological appearance of these cells, and only a fraction had a clear polymorphonuclear morphology. ${ }^{6}$ This heterogeneity is mimicked in human disease, where conflicting reports of neutrophil function obscure a singular defined role for these cells during Mtb infection. ${ }^{36}$ Of note, our observations in mice correlate with the reported accumulation of low-density granulocytes (LDGs) in patients with active TB. ${ }^{48}$ This as-of-yet poorly characterized granulocyte subpopulation increases in frequency with TB disease severity, ${ }^{48}$ and could represent a human analog to the Ly6G Mid cells described in our models. While heterogeneity complicates precise characterization of granulocyte subsets, our data indicate that Ly6G ${ }^{\text {Mid }}$ cells are abundant in susceptible mice, possess a lifespan at least as long as macrophages, poorly express MRTF-SRF-dependent antimicrobial genes, and become a major bacterial reservoir even in the context of an immune response capable of controlling Mtb in macrophages.

These data contribute to growing evidence of dysregulated granulocyte recruitment directly contributing to mycobacterial replication and $\mathrm{TB}^{6,23,36,48-50}$ The accumulation of granulocytes within multiple distinct murine models of susceptibility, along with neutrophil-associated transcriptional signatures predicting TB progression in humans, ${ }^{19-21}$ suggest a process representing a common pathway of disease progression downstream of distinct initiation events. As such, interrupting this process may represent a more promising interventional strategy than those targeting upstream mediators.

\section{MATERIALS AND METHODS}

Mice and infections

Wild-type, Nos2 $2^{-/-}, I L 10^{-/-}, C x C r 2^{-/-}$, and CD45.1 $1^{+/+}$(B6.SJL-

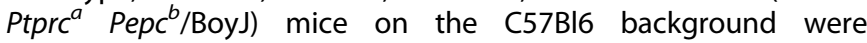
purchased from the Jackson Laboratory (Bar Harbor, ME), as were C3HeBFeJ mice. C57Bl6 Genista mice were a gift from Bernard Malissen at Aix-Marseille Université. 129SvPas mice were purchased from Charles River (Wilmington, MA). All mice were housed under specific pathogen-free conditions, in accordance with UMMS IACUC guidelines. Infections were carried out using PDIM-positive Mycobacterium tuberculosis strain H37Rv cultured 
a

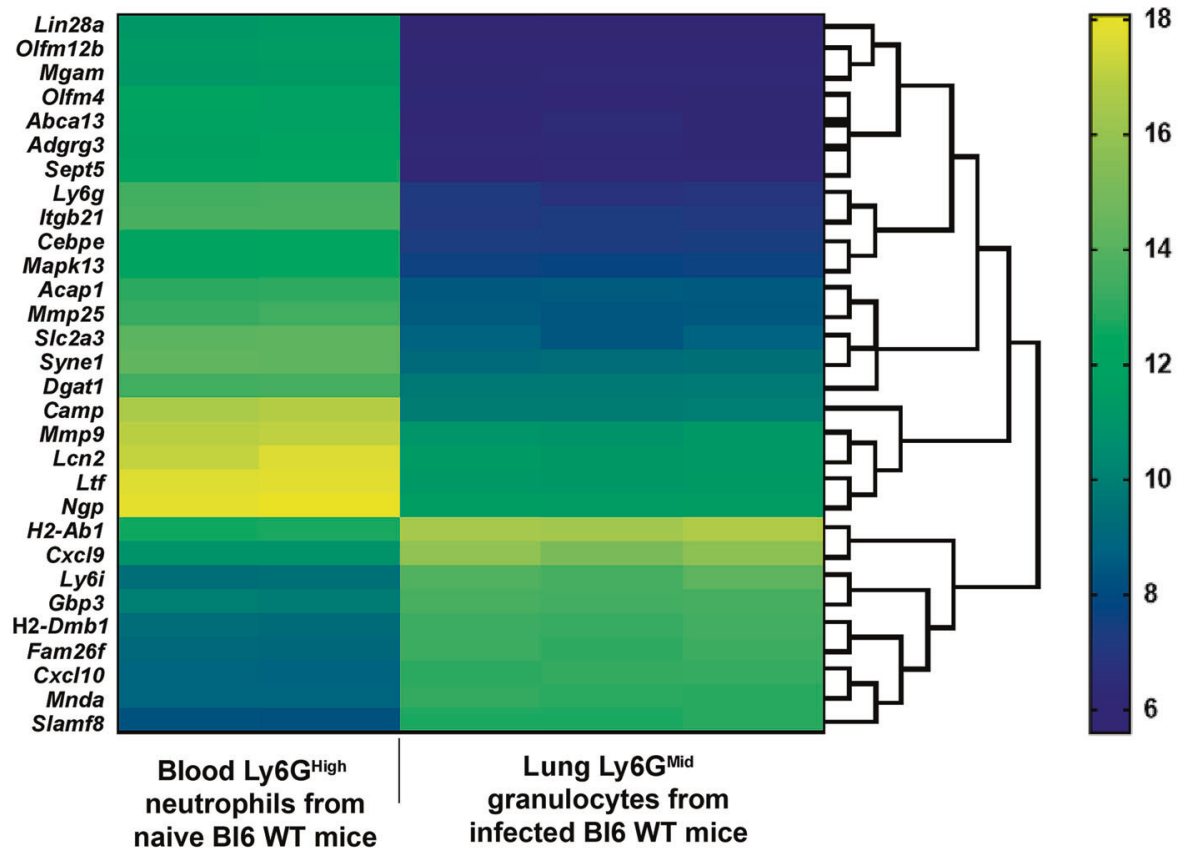

b
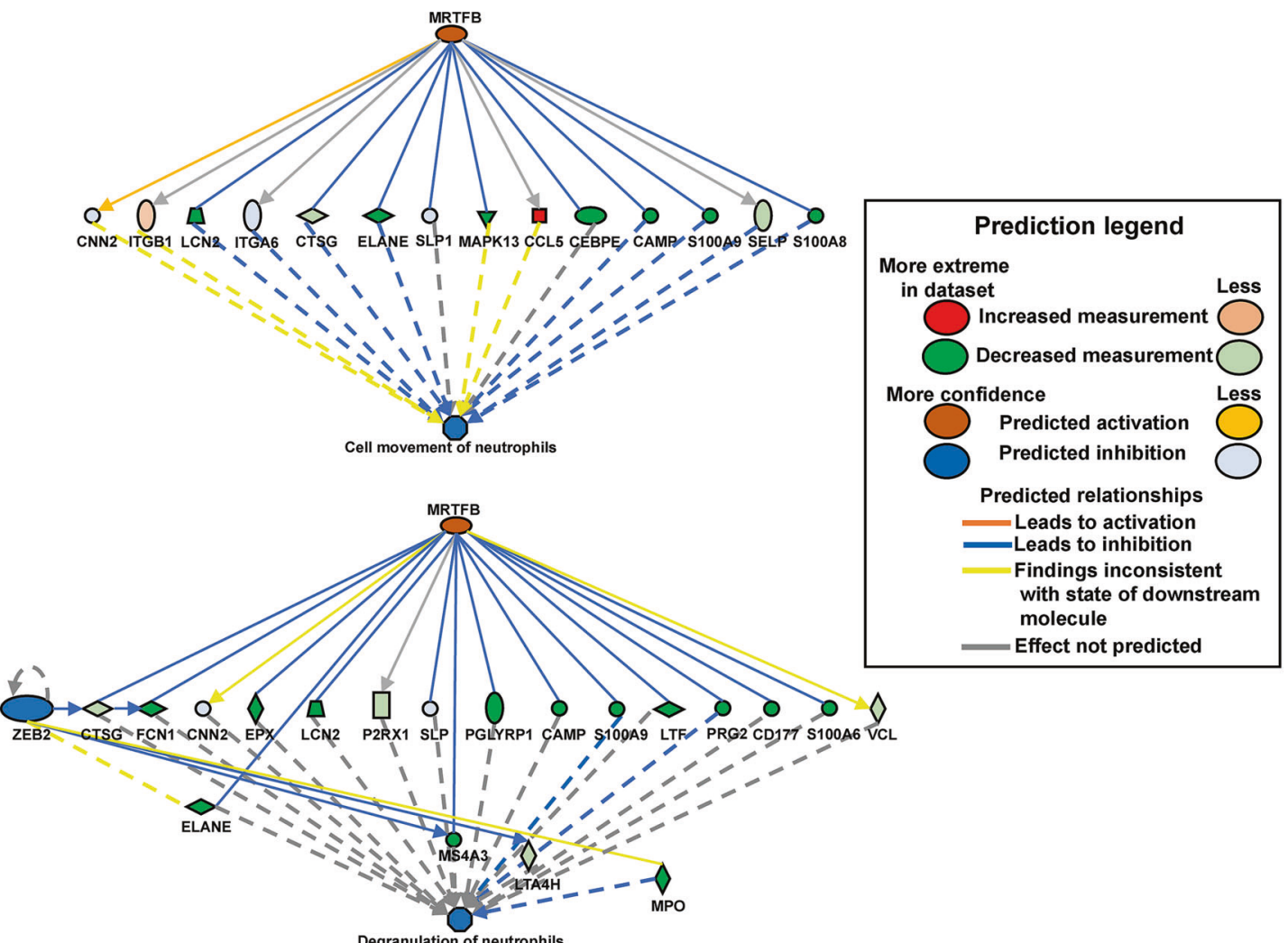

Fig. 6 Gene expression profile of Ly6G ${ }^{\text {Mid }}$ granulocytes indicates decreased antimicrobial function. a Bi-clustering heatmap visualizing the expression profile of the top 30 most differentially expressed genes, as sorted by adjusted $p$-value via plotting the log2 transformed expression values in each sample. b Pathway analysis of differentially expressed genes predicting activation or inhibition of upstream regulators in relation to functional neutrophil migration (top) or degranulation (bottom). $P$ value cutoff $\leq 0.01$. Ly6G ${ }^{\text {High }}$ peripheral blood neutrophils were recovered from uninfected BI6 WT mice $(N=2)$. Ly6G ${ }^{\text {Mid }}$ granulocytes were recovered from Bl6 WT mouse lungs at 32 days post infection $(N=3)$.

in 7 H9 medium containing $0.05 \%$ Tween 80 and OADC enrichment (Becton Dickinson/BD-Biosciences, San Jose, CA). Yellow fluorescent protein (msfYFP) expressing H37Rv were generated by transformation with plasmid PMV261, which constitutively expresses msfYFP under control of the hsp60 promoter. Murine aerosol infections of $\sim 200$ CFUs were carried out as described by Mishra et al. ${ }^{6}$ All mice were at least 6 weeks of age, and were sex and age matched within individual experiments. 


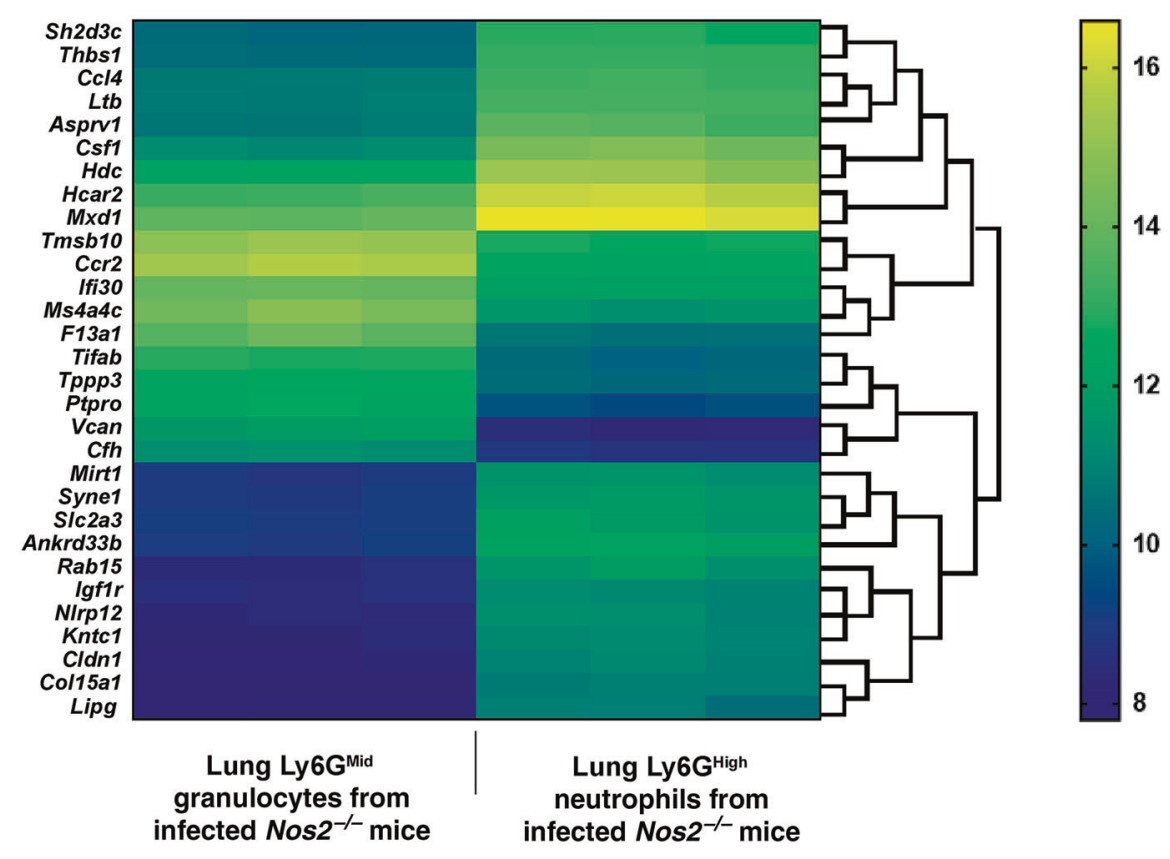

b

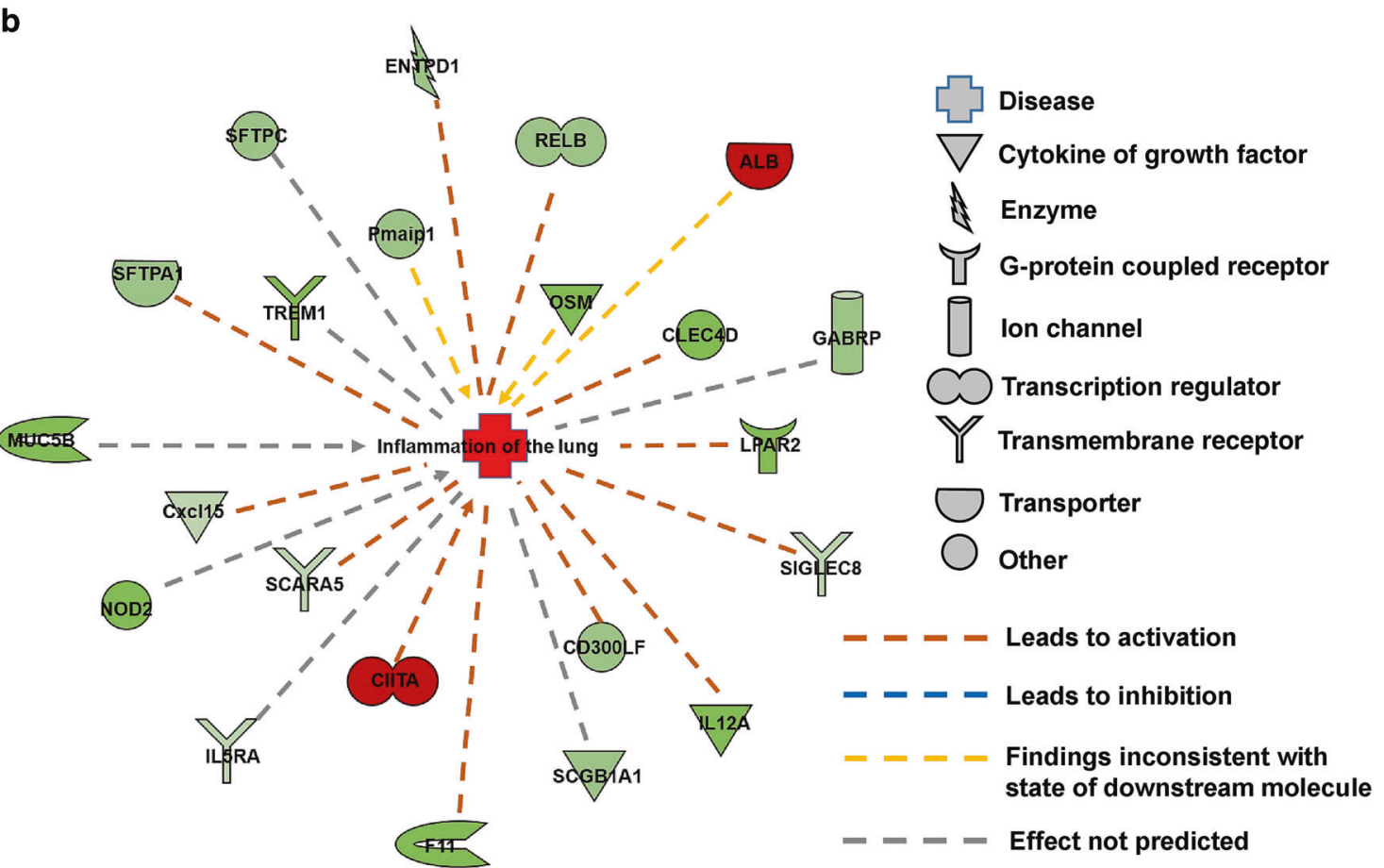

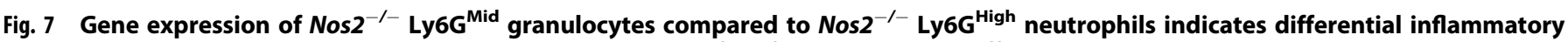
signaling. a Bi-clustering heatmap visualizing the expression profile of the top 30 most differentially expressed genes, as sorted by adjusted $p$ -

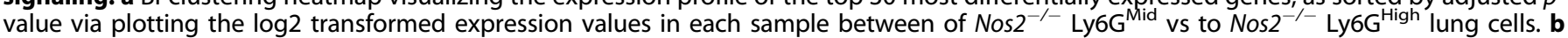
Differentially expressed genes were significantly enriched in the depicted inflammatory signaling pathway. $P$ value cutoff $\leq 0.01$. Cells were collected from mouse lungs at 32 days $\mathrm{PI} . \mathrm{N}=3$ mice per group.

\section{Flow cytometry}

Single-cell suspensions were prepared as described previously. ${ }^{6}$ Briefly, whole lungs were homogenized via Miltenyi (Bergisch Gladbach, Germany) GentleMACs dissociator in $5 \mathrm{~mL}$ RPMI plus Collagenase type IV/DNasel, then strained through a $40 \mu \mathrm{M}$ cell strainer. Analyses were performed on cells after 30 min staining at 4 ${ }^{\circ} \mathrm{C}$, using antibodies purchased from Biolegend (San Diego, California), and $20 \mathrm{~min}$ fixation using $4 \%$ paraformaldehyde or Cytoperm/Cytofix buffer (BD-Biosciences). For intracellular staining
(ICS), fixed cells were further stained with the indicated fluorophoreconjugated ICS antibody or isotype control overnight at $4{ }^{\circ} \mathrm{C}$, then washed and analyzed. For infections with fluorescent H37Rv, lung tissue was prepared as above, ${ }^{6}$ and compared against lung tissue from mice infected with non-fluorescent H37Rv.

Antibody-based cell depletion

Anti-Ly6G depleting antibody clone $1 \mathrm{~A} 8$ or isotype control $2 \mathrm{~A} 3$ (BioXcell, West Lebanon, $\mathrm{NH}$ ) was administered as described by 
Nandi et al. ${ }^{50}$ Ly6G $^{\text {Pos }}$ cell depletion was verified by FACS analysis of CD11b $b^{\text {High }}$ GR1 ${ }^{\text {Pos }}$ Ly6C $C^{\text {Neg }}$ cell populations (Supplementary Fig. 2a).

MDSC suppression assay

Ly6G ${ }^{\text {High }}$ or Ly6G ${ }^{\text {Mid }}$ cells were isolated from the lungs of WT C57BL6, Nos2 $2^{-1-}$, C3HeBFeJ, or 129SvPas mice at 28 days PI using the Miltenyi MDSC Isolation Kit. P25 CD4 ${ }^{\text {Pos }}$ T-cells were preactivated for $6 \mathrm{~h}$ in complete media containing interleukin-2 and CD3/CD28 Dynabeads ${ }^{\mathrm{TM}}$ (ThermoFisher Scientific, Waltham, MA) following the manufacturer's protocol, then incubated alone or in 1:1 co-culture with isolated granulocytes in 96-well plates $\left(10^{5}\right.$ total cells/well) at $37^{\circ} \mathrm{C}, 5 \% \mathrm{CO}_{2}$ for $24 \mathrm{~h}$. Culture supernatants were then removed, filter sterilized, and tested for total IFNy by ELISA.

in vivo half-life assay

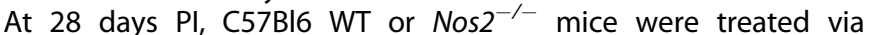
tracheal aspiration with $40 \mu \mathrm{L}$ of $1 \mathrm{mM}$ CellTrace $^{\mathrm{TM}}$ Violet dye (ThermoFisher Scientific) resuspended in dimethyl sulfoxide. Onehour post-treatment, a cohort from each genotype was euthanized, lungs harvested, and pulmonary tissue homogenized for Ab-staining. Subsequent cohorts were euthanized at $24 \mathrm{~h}, 48 \mathrm{~h}$, and $72 \mathrm{~h}$ post-treatment (Fig. 5a). Spleens were harvested, homogenized, and Ab-stained to verify dye restriction to the pulmonary compartment. Half-life values for individual cell populations were calculated by quantifying the number of dye ${ }^{\text {Pos }}$ cells within mice of each cohort (relative to untreated controls) using a flow cytometer, then calculating the linear regression of dye ${ }^{\text {Pos }}$ cell ablation over time using the formula: $\left(\left(\operatorname{Ln}(2)^{*} Z\right.\right.$ hours post-treatment)/(Ln(mean \# of dye ${ }^{\text {Pos }}$ cells at $1 \mathrm{~h}$ post-treatment/ mean \# of dye ${ }^{\text {Pos }}$ cells at $Z$ hours post-treatment))). The calculated cell half-lives were averaged within each genotype and cohort (biological replicates for each time point $=3$ mice per cohort per genotype, technical replicates for each time point $=9$ ). Any mouse with $<10 \%$ of total cells dye ${ }^{\text {Pos }}$ was discarded. All calculations were determined using 1-, 24-, and 48-h cohorts. No dye signal was observed at $72 \mathrm{~h}$ post-treatment.

\section{Analysis of YFP Unit distribution}

Pulmonary cells from msfYFP-Mtb infected mice were recovered, stained, and analyzed by flow cytometry as described above. ${ }^{6}$ The YFP fluorescence value of each individual event (relative to background) within specified populations was exported into CSV format. For each YFP ${ }^{\text {Pos }}$ population within each mouse, the lowest recorded YFP fluorescence value above background was set equal to 1 YFP Unit. All subsequent YFP ${ }^{\text {Pos }}$ events within the population were divided by this lowest positive value to generate bins. Histograms were generated with FlowPy graphing software, plotting the number of YFP ${ }^{\text {Pos }}$ cells falling within each respective bin.

\section{Sequencing and pathway analysis}

Approximately 1.25 million Ly6G ${ }^{\text {High }}$ or Ly6G ${ }^{\text {Mid }}$ cells were isolated from naïve mouse blood or Mtb infected mouse lungs using the Miltenyi MDSC Isolation Kit. RNA from cells in each test group was extracted, enriched for mRNA, fragmented, and randomly primed. First and second strand cDNA was synthesized and end-repaired by $5^{\prime}$ phosphorylation and $3^{\prime} \mathrm{dA}$-tailing. Adapters were ligated onto the CDNA fragments, which were then PCR enriched and sequenced via Illumina (San Diego, California) HiSeq, PE 2x150. Resulting reads were trimmed to remove possible adaptor sequence contamination and nucleotides with poor quality were discarded using Trimmomatic v.036. Trimmed reads were mapped to the Mus musculus GRCm28 reference genome using STAR aligner v.205.2b. Unique gene hit counts were calculated using featureCounts from the Subread package v.1.5.2, counting only those unique reads that fell within exon regions. Gene hit counts between test groups were compared using DESeq2. The Wald test generated $p$-values and $\log 2$ fold changes, with an adjusted $p$-value $<0.05$ and absolute $\log 2$ fold change $>1$ defining differentially expressed genes between comparisons. Predictive pathway analysis was performed using Quigen (Hilden, Germany) Ingenuity Pathway Analysis software, which compared significantly different -log2 gene expression values between test groups.

\section{Isolation of intracellular and extracellular $M t b$}

Lungs from C57BI6 WT or Nos2 $2^{-1-}$ mice were harvested at 14, 21, or 28 days post Mtb infection and mechanically homogenized with a Miltenyi GentleMACs dissociator. An aliquot of homogenate was plated in serial dilutions to determine total bacterial load, while the remainder was resuspended in PBS-T plus $70 \mathrm{mM}$ sucrose and centrifuged at $1000 \mathrm{rpm}$ for $5 \mathrm{~min}$ to pellet mammalian cells. An aliquot of supernatant was plated to quantify extracellular bacteria unassociated with mammalian cells. The remaining supernatant was treated with $50 \mu \mathrm{g} / \mathrm{mL}$ gentamicin for $20 \mathrm{~min}$, then plated as a control for the antibiotic killing of non-cell associated bacteria and the potential for non-pelleted mammalian cell contaminants. The cell pellet was resuspended in serum-free media and an aliquot was plated to quantify total cell-associated bacteria. The remaining cell pellet was halved. One-half was treated with gentamicin, as above, then washed $2 \times$ with cold PBS and lysed in $0.1 \%$ Triton$X 100$. Lysate was plated to quantify intracellular bacteria. The other half of the cell pellet was lysed as above, then treated with gentamicin and plated as a control for antibiotic killing of cellassociated bacteria. The sum of recovered extracellular CFUs plus total cell associated CFUs was compared against the total CFUs recovered from homogenate (prior to centrifugation) to verify that both intracellular and extracellular fractions were accounted for within each mouse (Supplementary Fig. 4a).

Generation of mixed bone marrow chimera mice B6.129S2(C)-CxCr2 ${ }^{\text {tm1Mwm }} / \mathrm{J}$ (CD45.2 $2^{+/+}$) mice were lethally irradiated with two doses of 600 rads. The following day, bone marrow from CD45.1 $1^{+/+}$WT mice, CD45.2 $2^{+/+}$CxCr2 $2^{-/-}$mice, or $\mathrm{CD} 45.2^{+/+} \mathrm{IL} 10^{-/-}$mice was isolated, red blood cells were lysed using Tris-buffered Ammonium Chloride (ACT), and remaining cells were quantified using a hemocytometer. CD45.1 ${ }^{+/+}$WT cells and CD45.2 $2^{+/+} \mathrm{CxCr} 2^{-/-}$cells, or, $1110^{-1-}$ cells and $\mathrm{CxCr} 2^{-/-}$cells, were mixed equally at a $1: 1$ ratio. $10^{7}$ cells from each respective mixture were then injected intravenously into separate lethally irradiated hosts (Fig. 3b). Reconstitution of host mice lasted 6-8 weeks with preventative sulfatrim treatment for the first 4 weeks. 50:50 chimerism was confirmed by bleeding reconstituted hosts, lysing red blood cells with ACT, and staining for CD $11 b^{\text {High }}$ blood myelocytes for expression of CD45.1, CD45.2, or CXCR2 (Fig. 3c).

\section{Cytokine measurements}

Murine cytokine concentrations in filter sterilized lung tissue lysate were quantified using commercial BD OptEIA ELISA kits or Eve Technologies (Calgary, AB Canada) 32-Plex Discovery Assay. All samples were normalized for total protein content.

Isolation of Ly6G ${ }^{\text {Pos }}$ cells from mouse lungs Ly6G ${ }^{\text {High }}$ cells or Ly6G Mid were isolated from WT C57BL6J, Nos2 $2^{-/-}$, $\mathrm{C} 3 \mathrm{H} 3 \mathrm{bFeJ}$, or 129SvPas mouse lung homogenates using the MDSC Isolation Kit from Miltenyi Biotec. Purity of Ly6G ${ }^{\text {Pos }}$ cell populations was tested by counterstaining with fluorophoreconjugated anti-GR1 (Clone RB6-8C5, Biolegend) and analyzing with a flow cytometer.

Aminoguanidine treatment of animals

Mice were treated ad libitum with $2.5 \%$ aminoguanidine over the course of infection as described previously, ${ }^{6}$ with an additional biweekly intraperitoneal injection of 500uL sterile water containing $2.5 \%$ aminoguanidine. 


\section{ACKNOWLEDGEMENTS}

Genista mice were a kind gift from Bernard Malissen at Aix-Marseille Université. We thank Dr. Hardy Kornfeld, Dr. Nuria Martinez, and Dr. Samuel Behar for conceptua discussions; Charlotte Reames, Michael Kiritsy, Michelle Bellerose, Caitlin Moss, Dr. Jason Yang, Dr. Lisa Lojek, Dr. Andrew Olive, and Kadamba Papavinasasundaram for technical assistance; and the biosafety level 3 and the flow cytometry core facilities at UMMS. This work was supported by the National Institutes of Health (grants to C.M.S. (Al32130) and to R.R.L. (F32Al120556).

\section{AUTHOR CONTRIBUTIONS}

R.R.L. and C.M.S. conceived of the study. R.R.L. performed all experiments, with contributions from C.M.S., B.B.M., and C.B. msfYFP-Mtb was constructed by C.B. Paper was written by R.R.L. and C.M.S. All authors approved the final paper.

\section{ADDITIONAL INFORMATION}

The online version of this article (https://doi.org/10.1038/s41385-020-0300-z) contains supplementary material, which is available to authorized users.

Competing interests: The authors declare no competing interests.

Publisher's note Springer Nature remains neutral with regard to jurisdictional claims in published maps and institutional affiliations.

\section{REFERENCES}

1. Canetti, G. The Tubercle Bacillus in the Pulmonary Lesion of Man: Histobacteriology and Its Bearing on the Therapy of Pulmonary Tuberculosis. (Springer Publishing Company, 1955).

2. Bustamante, J., Boisson-Dupuis, S., Abel, L. \& Casanova, J. L. Mendelian susceptibility to mycobacterial disease: Genetic, immunological, and clinical features of inborn errors of IFN- $\gamma$ immunity. Semin. Immunol. 26, 454-470 (2014).

3. Flynn, J. A. L. et al. An essential role for interferon $\gamma$ in resistance to mycobacterium tuberculosis infection. J. Exp. Med. 178, 2249-2254 (1993).

4. Cooper, A. M. et al. Disseminated tuberculosis in interferon $\gamma$ gene-disrupted mice. J. Exp. Med. 178, 2243-2247 (1993).

5. MacMicking, J. D., Taylor, G. A. \& McKinney, J. D. Immune control of tuberculosis by IFN- $\gamma$-inducible LRG-47. Science. 302, 654-659 (2003).

6. Mishra, B. B. et al. Nitric oxide prevents a pathogen-permissive granulocytic inflammation during tuberculosis. Nat. Microbiol. 2, 17072 (2017).

7. Zhang, G. et al. Allele-specific induction of IL-1 $\beta$ expression by C/EBP $\beta$ and PU.1 contributes to increased tuberculosis susceptibility. PLoS Pathog. 10, e1004426 (2014).

8. Mayer-Barber, K. D. \& Yan, B. Clash of the cytokine titans: counter-regulation of interleukin-1 and type i interferon-mediated inflammatory responses. Cell. Mol. Immunol. 14, 22-35 (2017).

9. Teles, R. M. B. et al. NIH Public Access. 339, 1448-1453 (2014).

10. Dorhoi, A. et al. Type I IFN signaling triggers immunopathology in tuberculosissusceptible mice by modulating lung phagocyte dynamics. Eur. J. Immunol. 44, 2380-2393 (2014).

11. Manzanillo, P. S., Shiloh, M. U., Portnoy, D. A. \& Cox, J. S. Mycobacterium tuberculosis activates the DNA-dependent cytosolic surveillance pathway within macrophages. Cell Host Microbe 11, 469-480 (2012).

12. Manca, C. et al. Hypervirulent M. tuberculosis W/Beijing strains upregulate type I IFNs and increase expression of negative regulators of the Jak-Stat pathway. J. Interferon Cytokine Res. 25, 694-701 (2005).

13. Zhang, G. et al. A proline deletion in IFNAR1 impairs IFN-signaling and underlies increased resistance to tuberculosis in humans. Nat. Commun. 9, 1-9 (2018).

14. Ji, D. X. et al. Type I interferon-driven susceptibility to Mycobacterium tuberculosis is mediated by IL-1Ra. Nat. Micobiology. 4, 2128-2135 (2019).

15. Xiong, $H$. et al. Distinct contributions of neutrophils and CCR2+ monocytes to pulmonary clearance of different Klebsiella pneumoniae strains. Infect. Immun. 83, 3418-3427 (2015).

16. Garvy, B. A. \& Harmsen, A. G. The importance of neutrophils in resistance to pneumococcal pneumonia in adult and neonatal mice. Inflammation 20, 499-512 (1996).

17. Chao-Tsung, L. Y. et al. Neutrophils exert protection in the early tuberculosis granuloma by oxidative killing of mycobacteria phagocytosed from infected macrophages. Cell Host Microbe 112, 301-312 (2012).

18. Dallenga, T. \& Schaible, U. E. Neutrophils in tuberculosis-first line of defence or booster of disease and targets for host-directed therapy? Pathog. Dis. 74, ftw012 (2016).
19. Eum, S. Y. et al. Neutrophils are the predominant infected phagocytic cells in the airways of patients with active pulmonary TB. Chest 137, 122-128 (2010).

20. Berry, M. P. R. et al. An interferon-inducible neutrophil-driven blood transcriptional signature in human tuberculosis. Nature 466, 973-977 (2010).

21. Liu, Q. et al. Proteomic profiling for plasma biomarkers of tuberculosis progression. Mol. Med. Rep. 18, 1551-1559 (2018).

22. Nouailles, G. et al. CXCL5-secreting pulmonary epithelial cells drive destructive neutrophilic inflammation in tuberculosis. J. Clin. Invest. 124, 1268-1282 (2014).

23. Kimmey, J. M. et al. Unique role for ATG5 in neutrophil-mediated immunopathology during M. tuberculosis infection. Nature 528, 565-569 (2015).

24. Kruger, P. et al. Neutrophils: between host defence, immune modulation, and tissue injury. PLoS Pathog. 11, e1004651 (2015).

25. Gideon, H. P., Phuah, J., Junecko, B. A. \& Mattila, J. T. Neutrophils express pro- and anti-inflammatory cytokines in granulomas from Mycobacterium tuberculosisinfected cynomolgus macaques. Mucosal Immunol. 12, 1370-1381 (2019).

26. Denis, M. \& Ghadirian, E. IL-10 neutralization augments mouse resistance to systemic Mycobacterium avium infections. J. Immunol. 151, 5425-5430 (1993).

27. Knaul, J. K. et al. Lung-residing myeloid-derived suppressors display dual functionality in murine pulmonary tuberculosis. Am. J. Respir. Crit. Care Med. 190, 1053-1066 (2014).

28. Tsiganov, E. N. et al. Gr-1 dim CD11b + immature myeloid-derived suppressor cells but not neutrophils are markers of lethal tuberculosis infection in mice. J. Immunol. 192, 4718-4727 (2014).

29. Magcwebeba, T., Dorhoi, A. \& du Plessis, N. The emerging role of myeloid-derived suppressor cells in tuberculosis. Front. Immunol. 10, 917 (2019).

30. Lowe, D. M., Redford, P. S., Wilkinson, R. J., O'Garra, A. \& Martineau, A. R. Neutrophils in tuberculosis: Friend or foe? Trends Immunol. 33, 14-25 (2012).

31. Eruslanov, E. B. et al. Neutrophil responses to mycobacterium tuberculosis infection in genetically susceptible and resistant mice. Infect. Immun. 73, 1744-1753 (2005).

32. Marzo, E. et al. Damaging role of neutrophilic infiltration in a mouse model of progressive tuberculosis. Tuberculosis 94, 55-64 (2014).

33. Ordoñez-Rueda, D. et al. A hypomorphic mutation in the Gfi 1 transcriptional repressor results in a novel form of neutropenia. Eur. J. Immunol. 42, 2395-2408 (2012).

34. Janssen, W. J., Bratton, D. L., Jakubzick, C. V. \& Henson, P. M. Myeloid cell turnover and clearance. Microbiol. Spectr. 4, MCHD-0005-2015 (2016).

35. Garley, M. \& Jabłońska, E. Heterogeneity Among Neutrophils. Arch. Immunol. Ther. Exp. (Warsz.). 66, 21-30 (2018).

36. Lyadova, I. V. Neutrophils in tuberculosis: heterogeneity shapes the way? Mediators Inflamm. 2017, 8619307 (2017).

37. Jena, P. et al. Azurophil granule proteins constitute the major mycobactericidal proteins in human neutrophils and enhance the killing of Mycobacteria in macrophages. PLoS ONE 7, 1-13 (2012).

38. Saiga, H. et al. Lipocalin 2-Dependent inhibition of mycobacterial growth in alveolar epithelium. J. Immunol. 181, 8521-8527 (2008).

39. Actor, J. K. Lactoferrin: a modulator for immunity against tuberculosis related granulomatous pathology. Mediators Inflamm. 2015, 409596 (2015).

40. Sonawane, A. et al. Cathelicidin is involved in the intracellular killing of mycobacteria in macrophages. Cell. Microbiol. 13, 1601-1617 (2011).

41. Liu, Y., Nonnemacher, M. R. \& Wigdahl, B. CCAAT/enhancer-binding proteins and the pathogenesis of retrovirus infection. Future Microbiol. 4, 299-321 (2009).

42. Taylor, A. et al. SRF is required for neutrophil migration in response to inflammation. Blood 123, 3027-3036 (2014).

43. Bruhn, K. W., Dekitani, K., Nielsen, T. B., Pantapalangkoor, P. \& Spellberg, B. Ly6G mediated depletion of neutrophils is dependent on macrophages. Results Immunol. 6, 5-7 (2016).

44. Deniset, J. F., Surewaard, B. G., Lee, W. Y. \& Kubes, P. Splenic Ly6Ghigh mature and Ly6Gint immature neutrophils contribute to eradication of $\mathrm{S}$. pneumoniae. J. Exp. Med. 214, 1333 (2017).

45. Elghetany, M. T. Surface antigen changes during normal neutrophilic development: a critical review. Blood Cells, Mol. Dis. 28, 260-274 (2002).

46. Evrard, M. et al. Developmental analysis of bone marrow neutrophils reveals populations specialized in expansion, trafficking, and effector functions. Immunity 48, 364-379 (2018).

47. MacNamara, K. C. et al. Infection-induced Myelopoiesis during intracellular bacterial infection is critically dependent upon IFN- $\gamma$ signaling. J. Immunol. 186, 1032-1043 (2011).

48. Deng, Y. et al. Low-density granulocytes are elevated in mycobacterial infection and associated with the severity of tuberculosis. PLOS ONE 11, e0153567 (2016).

49. Warren, E., Teskey, G. \& Venketaraman, V. Effector Mechanisms of Neutrophils within the Innate Immune System in Response to Mycobacterium tuberculosis Infection. J. Clin. Med. 6, 1-9 (2017).

50. Nandi, B. \& Behar, S. M. Regulation of neutrophils by interferon- $\gamma$ limits lung inflammation during tuberculosis infection. J. Exp. Med. 208, 2251-2262 (2011). 